\title{
Differential Proteome Profiling Using iTRAQ in Microalbuminuric and Normoalbuminuric Type 2 Diabetic Patients
}

\author{
Jonghwa Jin, ${ }^{1}$ Yun Hyi Ku, ${ }^{2}$ Yikwon Kim, ${ }^{1}$ Yeonjung Kim, ${ }^{1}$ Kyunggon Kim, ${ }^{1}$ Ji Yoon Lee, ${ }^{3}$ \\ Young Min Cho, ${ }^{2}$ Hong Kyu Lee, ${ }^{2}$ Kyong Soo Park, ${ }^{2,4}$ and Youngsoo Kim ${ }^{1}$ \\ ${ }^{1}$ Department of Biomedical Sciences, Seoul National University College of Medicine, 28 Yongon-Dong, Seoul 110-799, Republic of Korea \\ ${ }^{2}$ Department of Internal Medicine, Seoul National University College of Medicine, 28 Yongon-Dong, Seoul 110-799, Republic of Korea \\ ${ }^{3}$ National Instrumentation Center for Environmental Management, Seoul National University, Seoul 151-921, Republic of Korea \\ ${ }^{4}$ Genome Research Center for Diabetes and Endocrine Disease, Seoul National University College of Medicine, 28 Yongon-Dong, \\ Seoul 110-799, Republic of Korea
}

Correspondence should be addressed to Kyong Soo Park, kspark@snu.ac.kr and Youngsoo Kim, biolab@snu.ac.kr

Received 2 July 2011; Revised 27 October 2011; Accepted 22 November 2011

Academic Editor: K. Herbert

Copyright (C) 2012 Jonghwa Jin et al. This is an open access article distributed under the Creative Commons Attribution License, which permits unrestricted use, distribution, and reproduction in any medium, provided the original work is properly cited.

\begin{abstract}
Diabetic nephropathy $(\mathrm{DN})$ is a long-term complication of diabetes mellitus that leads to end-stage renal disease. Microalbuminuria is used for the early detection of diabetic renal damage, but such levels do not reflect the state of incipient DN precisely in type 2 diabetic patients because microalbuminuria develops in other diseases, necessitating more accurate biomarkers that detect incipient DN. Isobaric tags for relative and absolute quantification (iTRAQ) were used to identify urinary proteins that were differentially excreted in normoalbuminuric and microalbuminuric patients with type 2 diabetes where 710 and 196 proteins were identified and quantified, respectively. Some candidates were confirmed by 2-DE analysis, or validated by Western blot and multiple reaction monitoring (MRM). Specifically, some differentially expressed proteins were verified by MRM in urine from normoalbuminuric and microalbuminuric patients with type 2 diabetes, wherein alpha-1-antitrypsin, alpha-1-acid glycoprotein 1, and prostate stem cell antigen had excellent AUC values $(0.849,0.873$, and 0.825 , resp.). Moreover, we performed a multiplex assay using these biomarker candidates, resulting in a merged AUC value of 0.921. Although the differentially expressed proteins in this iTRAQ study require further validation in larger and categorized sample groups, they constitute baseline data on preliminary biomarker candidates that can be used to discover novel biomarkers for incipient DN.
\end{abstract}

\section{Introduction}

Diabetes mellitus is a chronic disease with potentially devastating complications. For example, diabetes mellitus is associated with macrovascular complications, such as cardiovascular and cerebrovascular diseases, and microvascular complications, including diabetic nephropathy (DN) and retinopathy [1]. DN is a long-term complication of diabetes that is caused by specific renal structural alterations, such as mesangium expansion due to the progressive accumulation of extracellular matrix (ECM), and by functional losses, such as elevated glomerular basement membrane (GBM) permeability [2].
DN occurs in $15 \%$ to $25 \%$ of type 1 diabetic patients and $30 \%$ to $40 \%$ of type 2 diabetic patients [3] and accounts for approximately one-half of all new cases of end-stage renal disease (ESRD). Furthermore, ESRD has a 5-year survival rate of only $21 \%$ [4]. Because the progression of ESRD in $\mathrm{DN}$ is irreversible, the early diagnosis of $\mathrm{DN}$ is necessary to prevent or delay progression to ESRD [5]. Microalbuminuria represents a potentially reversible incipient stage of nephropathy and is used as a noninvasive index for the detection of diabetic renal disease. Microalbuminuria is defined as a state in which abnormal amounts of albumin are excreted in urine $(30-300 \mathrm{mg} / 24 \mathrm{~h}$ versus $<30 \mathrm{mg} / 24 \mathrm{~h}$ in normoalbuminuria) $[5,6]$. 
The use of microalbuminuria to predict incipient DN, particularly in type 2 diabetic patients, is limited for several reasons [7]: the microalbuminuric state also predicts cardiovascular disease in diabetic and nondiabetic individuals [8, 9], and it is associated with inflammation and hypertension [5]. Consequently, the likelihood of detecting nondiabetic renal disease or normal glomerular structure is observed with microalbuminuria patients [10]. Thus, more accurate biomarkers for incipient DN in type 2 diabetic patients are required that can differentiate incipient DN from other conditions in microalbuminuria patients, including cardiovascular disease, inflammation, and hypertension.

Recently, to compare DN patients with non-DN patients, proteomic technologies have been developed to identify urinary marker candidates that are associated with the development of DN. Various proteomic approaches have been used for this purpose, including 2-DE, 2-DE DIGE, and SELDITOF $[5,11,12]$. However, because many studies have focused on restricted sets of targeted proteins, alterations in comprehensive urinary protein profiles in type 2 diabetes have not been monitored. In particular, SELDI-TOF has been shown to be a valuable technology for urinary proteomic analysis, but the absolute identification of differentially excreted proteins remains challenging [13].

To scan a comprehensive differential proteome for preliminary DN candidate biomarkers, we used a 4-plex isobaric tag for relative and absolute quantification (iTRAQ, 4-plex), allowing us to identify and quantify proteins in up to 4 samples [14]. The advantages of iTRAQ include whole labeling of representative or pooled samples, comparatively high throughput, and retention of posttranslational modification (PTM) data; one of its shortcomings is that it cannot be applied easily to a large collection of individual clinical samples due to reagent cost and the required mass spectrometry effort [15]. To date, iTRAQ has been applied to a variety of sample sets, including E. coli, mammalian cells, yeast, plant cells, and human biological fluids [16-22].

Therefore, in this study, we used iTRAQ to identify and quantify differentially excreted urinary proteins in microalbuminuric versus normoalbuminuric type 2 diabetic patients and investigate the associations that would reflect the progress of DN. Afterward, those differentially excreted urinary proteins have been confirmed by 2-DE, followed by MALDITOF/TOF, or validated by Western blot and MRM.

\section{Materials and Methods}

2.1. Urine Sample Preparation. Type 2 diabetic subjects (age $\geq 40$ years) with or without microalbuminuria who were patients at the Diabetes Center of Seoul National University Hospital, Seoul, Republic of Korea, were enrolled in 2006. Microalbuminuria patients were randomly selected out of these outpatients, whereas normoalbuminuric patients were selected to be matched to age, sex, body mass index (BMI), and DM duration with microalbuminuric patients.

Forty-three subjects with diabetic retinopathy and persistent microalbuminuria formed the microalbuminuria group (MA). Persistent microalbuminuria was defined as an albumin: creatinine ratio (ACR) between 30 and $300 \mathrm{mg} / \mathrm{g}$ in
2 urine samples that were taken over 3 months. The normoalbuminuria group (NA) comprised subjects who had no diabetic retinopathy, did not use angiotensin inhibitors or angiotensin receptor blockers that lowered albuminuria, and showed no microalbuminuria in their urine in the past year (urinary albumin $<30 \mathrm{mg} / \mathrm{g}$ creatinine). Forty-three subjects formed the NA group.

There were no significant differences in age, sex, body mass index, or diabetes mellitus duration between the 2 study groups. Subjects with hematuria, uncontrolled hypertension (blood pressure $\geq 140 / 90 \mathrm{~mm} \mathrm{Hg}$ ), uncontrolled hyperglycemia (glycated hemoglobin A1c $\geq 8.5 \%$ ), urinary tract infection, acute febrile illness, congestive heart failure, or malignancy were excluded. Individuals who were receiving peroxisome proliferator-activated receptor gamma agonists were also excluded. Midstream urine of spot urine samples were collected in sterile $50-\mathrm{mL}$ tubes that contained $50 \mu \mathrm{L}$ $0.1 \mathrm{mM}$ PMSF (serine protease inhibitor) and $500 \mu \mathrm{L} 1 \mathrm{mM}$ sodium azide from 86 patients and were stored at $-80^{\circ} \mathrm{C}$ until use. Informed consent was obtained from all subjects after obtaining approval for the study from the Institutional Review Board at Seoul National University Hospital.

Urine albumin and creatinine were measured in spot urine samples by immunoturbidimetric method using the TIA Micro Alb Kit (Nittobo, Tokyo, Japan) and enzymatic creatinine assay (Roche, Mannheim, Germany), respectively, on a Hitachi 7170 autoanalyzer (Hitachi, Tokyo, Japan).

For the ITRAQ and 2-DE experiments, pooled urine samples, based on average albumin-to-creatinine ratios, were used; the clinical characteristics of the study subjects are summarized in Table 1. Because the protein concentration of each urine sample varied widely, depending on the urine volume in the morning, equal amounts of total protein from each patient were pooled to prepare the urine samples (NA1NA4 and MA1-MA4).

To prepare the protein samples, approximately $50 \mathrm{~mL}$ aliquots of normoalbuminuric and microalbuminuric urine were centrifuged at $3000 \mathrm{~g}$ for $30 \mathrm{~min}$ at $4^{\circ} \mathrm{C}$. Supernatants were filtered through a $0.22 \mu \mathrm{m}$ MILLEX GP membrane (Millipore, Carrigtwohill, Cork, Ireland) and concentrated to $3 \mathrm{~mL}$ in an Amicon ultrafiltration cell (YM2, $3 \mathrm{kDa}$ MW cutoff, Millipore). The concentrated urine samples were then desalted by dialysis twice using a Slide-A-Lyzer dialysis cassette kit (3.5 kDa, Pierce, Rockford, ILUSA) against 1000 volumes of distilled water, containing $0.1 \mathrm{mM}$ PMSF (serine protease inhibitor) and $1 \mathrm{mM} \beta-\mathrm{ME}$, at $4^{\circ} \mathrm{C}$. Proteins in the dialyzed urine were precipitated with 5 volumes of acetone for $4 \mathrm{hrs}$ at $-20^{\circ} \mathrm{C}$, and the resulting pellets were washed 3 times with cold acetone; the supernatants were discarded.

2.2. Labeling with iTRAQ Reagents. Aliquots of $100 \mu \mathrm{g}$ of protein were reduced, alkylated, digested, and labeled according to the manufacturer's instructions (Applied Biosystems, Foster City, CA, USA). Briefly, $1 \mu \mathrm{L}$ of denaturant (2\% SDS) and $1 \mu \mathrm{L}$ of reducing reagent $(50 \mathrm{mM}$ tris-[2-carboxyethyl] phosphine) were added to each sample and incubated for $1 \mathrm{hr}$ at $60^{\circ} \mathrm{C}$. Each sample was allowed to cool at room temperature, and $1 \mu \mathrm{L}$ of cysteine blocking reagent $(200 \mathrm{mM}$ methyl methanethiosulfonate (MMTS) in isopropanol) was 


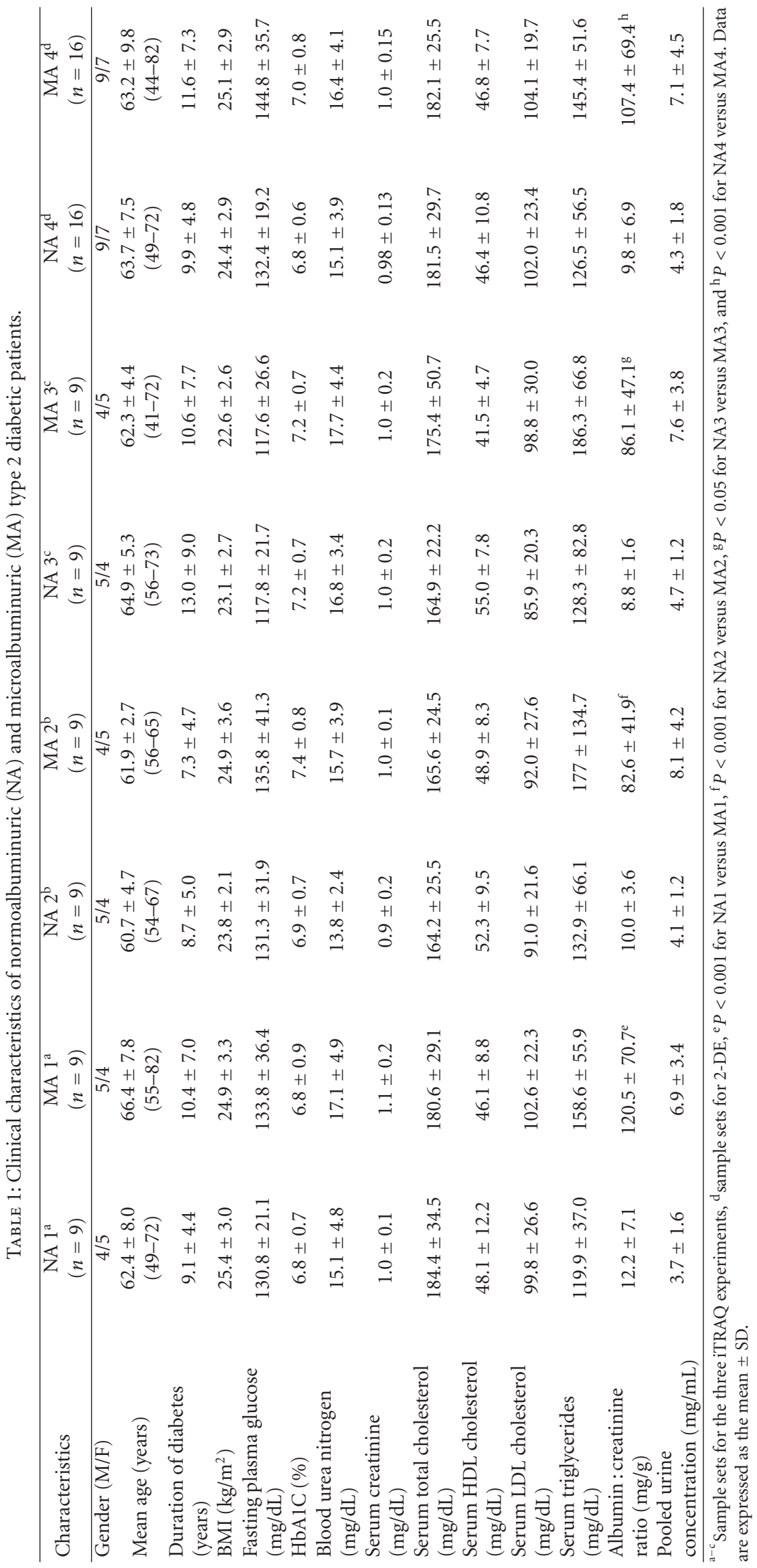


added and incubated for $20 \mathrm{~min}$ at room temperature. The tubes were digested with trypsin (Promega, Madison, WI, USA) at a protein-to-enzyme ratio of $10: 1$ at $37^{\circ} \mathrm{C}$ overnight, and the contents of one vial of iTRAQ reagent, dissolved in $70 \mu \mathrm{L}$ of ethanol, were added to each peptide mixture and incubated for $1 \mathrm{hr}$ at room temperature.

In this study, 3 iTRAQ experiments were performed. The detailed ITRAQ labeling strategy is summarized for the specified NA/MA urine samples in Figure 1 and Table 1; iTRAQ Experiments 1, 2, and 3 were performed for labeling (a) and (b), (c) and (d), and (e), respectively. Each normoalbuminuric peptide was labeled with iTRAQ reagents 114,115 , and 116 , and the microalbuminuric peptide was labeled with iTRAQ reagents 115 and 117 (Figure 1). The 2 sample sets (microalbuminuric and normoalbuminuric) were combined and dried. To analyze the proteome quantitatively using iTRAQ labeling, we determined the labeling efficiency, as described [23]; the number of possible labeling sites (the $\mathrm{N}$-termini of all peptides and lysine side chains) in 21,610 peptides were compared manually with that of completely labeled sites, represented by the Pro GroupTM Algorithm in ProteinPilot.

\subsection{Strong Cation Exchange Chromatographic Fractionation.} iTRAQ-labeled samples were subjected to LC-MS/MS at the National Instrumentation Center for Environmental Management, Seoul National University, and fractionated using strong cation exchange (SCX) chromatography, as follows. Dried samples were reconstituted in $500 \mu \mathrm{L}$ of buffer $\mathrm{A}$ $(25 \% \mathrm{v} / \mathrm{v}$ acetonitrile $(\mathrm{ACN})$ and $5 \mathrm{mM}$ ammonium formate, adjusted to $\mathrm{pH} 2.7$ with formic acid) and loaded onto a PolySULFOETHYL A column $(4.6 \mathrm{~mm}$ id $\times 100 \mathrm{~mm}, 5 \mu \mathrm{m}$, $200 \AA$ A PolyLC, Columbia, MD, USA) in a HP1100 series HPLC (Agilent Technologies, Palo Alto, CA, USA). The column was equilibrated for $5 \mathrm{~min}$ in buffer $\mathrm{A}$, and the peptides were eluted using a gradient of $0 \%$ to $30 \%$ buffer B $(25 \% \mathrm{v} / \mathrm{v}$ $\mathrm{ACN}$ and $1 \mathrm{M}$ ammonium formate [pH 3] with formic acid) over $80 \mathrm{~min}$ and $30 \%$ to $90 \%$ buffer B for $40 \mathrm{~min}$ at a flow rate of $0.7 \mathrm{~mL} / \mathrm{min}$. Absorbance was monitored at $280 \mathrm{~nm}$, and the fractions were collected every 2 min after injection.

2.4. LC-MS/MS Analysis. Fractions were reconstituted in solvent A and injected into an LC-ESI-MS/MS system. LCMS/MS was performed using an integrated system, which consisted of an autosampler switching pump and a micropump (Tempo Nano LC system; Applied Biosystems) with a hybrid quadrupole-TOF LC-MS/MS spectrometer (QStar Elite; Applied Biosystems) that was equipped with a nanoelectrospray ionization source (Applied Biosystems) and fitted with a $10 \mu \mathrm{m}$ fused silica emitter tip (New Objective, Woburn, MA, USA).

Peptides were first trapped on a Zorbax 300SB-C18 trap column $(300 \mu \mathrm{m}$ id $\times 5 \mathrm{~mm}, 5 \mu \mathrm{m}, 100 \AA$ A Agilent Technologies), washed for $10 \mathrm{~min}$ with $98 \%$ solvent A (water/ACN [98:2 v/v] and $0.1 \%$ formic acid) and $2 \%$ solvent B (water/ ACN $[2: 98 \mathrm{v} / \mathrm{v}]$ and $0.1 \%$ formic acid) at a flow rate of $10 \mu \mathrm{L} / \mathrm{min}$, and separated on a Zorbax 300SB-C18 capillary column $(75 \mu \mathrm{m}$ id $\times 150 \mathrm{~mm}, 3.5 \mu \mathrm{m}, 100 \AA)$ at a flow rate of $300 \mathrm{~nL} / \mathrm{min}$. The LC gradient was run at $2 \%$ to $35 \%$ solvent
B over $120 \mathrm{~min}$ and from $35 \%$ to $90 \%$ over $10 \mathrm{~min}$, followed by $90 \%$ solvent B for $15 \mathrm{~min}$, and finally $5 \%$ solvent B for $35 \mathrm{~min}$. The resulting peptides were electrosprayed through a coated silica tip (New Objective) at an ion spray voltage of $2300 \mathrm{eV}$.

For data acquisition, the mass spectrometer was set in the positive ion mode at a selected mass range of $400-1600 \mathrm{~m} / \mathrm{z}$ for a $1 \mathrm{sec}$ TOF-MS survey scan to detect precursor ions. The 5 most abundant peptides (count $>20$ ) with charge states of +2 to +4 were selected to perform the information-dependent acquisition (IDA) of MS/MS data. Once selected, the precursor ions were dynamically excluded for $60 \mathrm{sec}$ at a mass tolerance of $100 \mathrm{ppm}$.

2.5. Data Analysis. Data file processing, protein identification, and relative abundance quantification were performed using ProteinPilot v.2.0.1 (Applied Biosystems; MDS-Sciex, Concord, Canada) and the Paragon algorithm [24]. Database searches were performed against the Celera human database (human KBMS 5.0, 2005-03-02; a total of 187,748 entries provided by Applied Biosystems). The search parameters used were: a peptide and fragment ion mass tolerance of $0.2 \mathrm{Da} ; 1$ missed trypsin cleavage; fixed cysteine modification by MMTS; variable oxidation of methionine; and iTRAQ labeling of the N-termini of peptides and lysine side chain residues.

The confidence threshold for protein identification was an unused ProtScore $>1.3$ (95\% confidence interval). ProteinPilot computes a percentage confidence that reflects the probability that a hit is a false positive; thus, at the $95 \%$ confidence level, the false positive identification rate is approximately $5 \%[24,25]$. Although this program automatically accepts all peptides that have a confidence level $>1 \%$, only proteins with at least 1 peptide that had a confidence level $>95 \%$ were initially recorded. At these low confidence levels, peptides do not identify a single protein by themselves but, rather, support protein identification in the presence of other peptides [24, 25]. Quantification results were reported only when the error factor $(\mathrm{EF})$ was $<2$, which indicates a standard deviation of quantification $<20 \%$.

2.6. GO Ontology Analysis. The "biological process" and "molecular function" classifications were analyzed using PANTHER ID numbers (http://www.pantherdb.org/), provided in the ProteinPilot output when a Celera human database is used. To construct a graphical representation of differentially excreted proteins, MultiExperiment Viewer (Version 4.3) was used, allowing us to generate a "heatmap" of differentially excreted proteomes (http://www.tm4.org/mev/).

2.7. 2-DE Urinary Proteome and PMF Analysis. Urine samples were pooled from 16 type 2 diabetic patients with normoalbuminuria and 16 type 2 diabetic patients with microalbuminuria. The characteristics of the pooled urine samples for 2-DE are shown in Figure 1 and Table 1. For the PMF analysis, a MALDI-TOF/TOF mass spectrometer (ABI 4700 Proteomics Analyzer, Applied Biosystems) was used as described in our previous papers [26, 27]. 

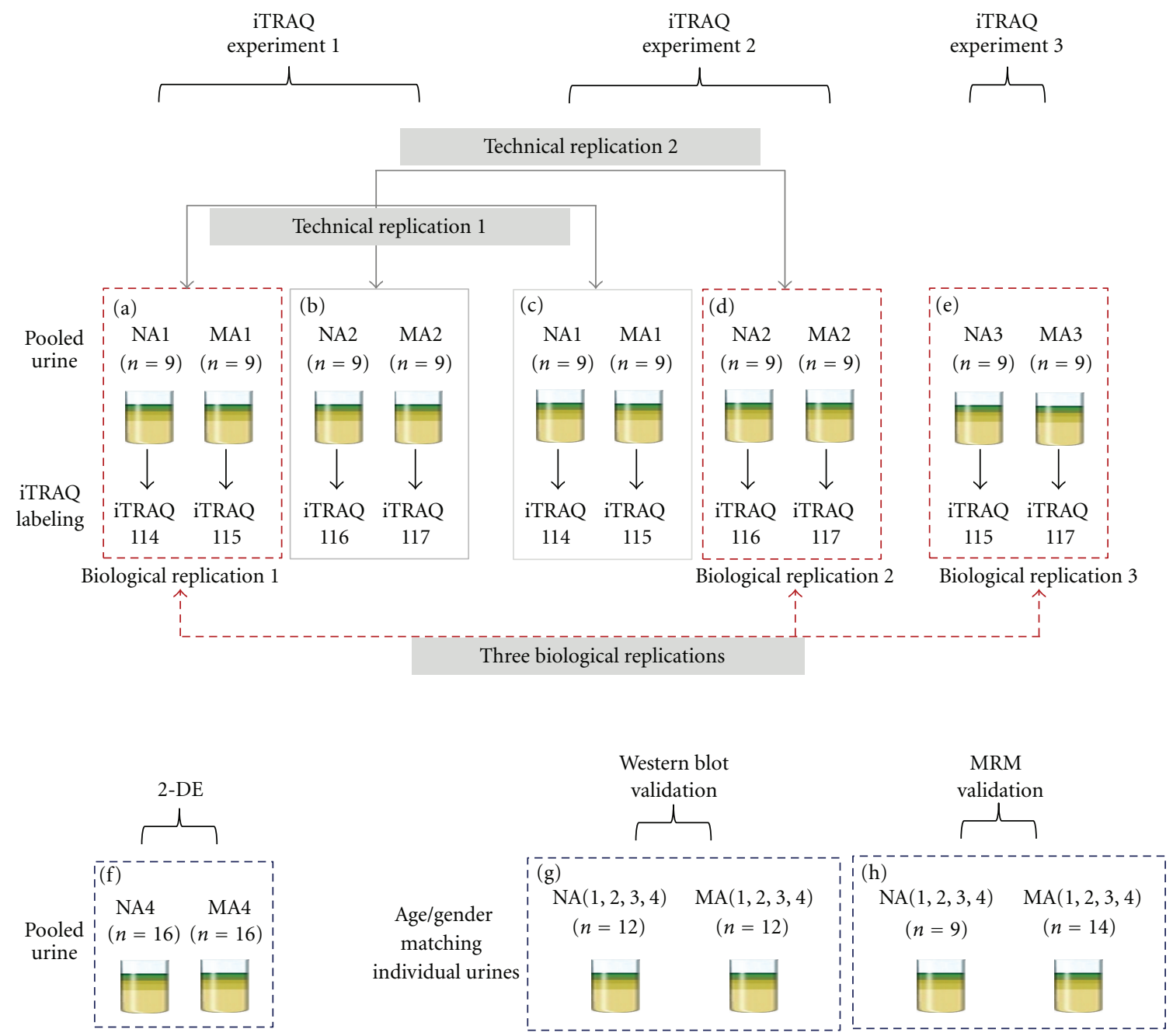

FIGURE 1: Workflow of ITRAQ, 2-DE, Western blot, and MRM of the urinary proteome. For analysis of the urinary proteome, 3 iTRAQ experiments were performed, 2-DE, Western blot, and MRM were conducted to confirm and validate the iTRAQ results. iTRAQ experiments 1, 2, and 3 were performed, labeled (a) and (b), (c) and (d), and (e), respectively, wherein 3 biological replicates (labeled (a), (d), and (e), resp.), technical replicate 1 (labeled (a) and (c)), and technical replicate 2 (labeled (b) and (d)) were performed in microalbuminuric versus normoalbuminuric urine. 2-DE, Western blot, and MRM analysis of the urinary proteome were conducted using labeled (f), (g), and (h), respectively.

2.8. Western Blot Analysis. Twenty-four urine samples that were matched for gender and age (NA: 6 females and 6 males, and MA: 6 females; 6 males) were selected from the urine sample groups (NA1-NA4 and MA1-MA4, resp.) and subjected to Western blot validation of the 6 representative candidates from the iTRAQ experiments (Figures 1 and 6, and Table 1). The primary antibodies were directed against transferrin (1:500, AbFrontier, Seoul, Korea), ceruloplas$\min (1: 1000$, AbFrontier), $\alpha 1$-antitrypsin $(1: 1000$, AbFrontier), vitamin D-binding protein $(1: 1000$, AbFrontier), $\alpha 1$ acid glycoprotein $(1: 2000$, AbFrontier), and haptoglobin ( $1: 1000$, AbFrontier).

2.9. Candidate Validation Using Multiple Reaction Monitoring. In addition to Western blot, multiple reaction monitoring (MRM) was performed to verify the candidate biomarkers using $9 \mathrm{NA}$ and $14 \mathrm{MA}$ urine samples from the urine sample groups (NA1-NA4 and MA1-MA4, resp.)
(Figure 1 and Table 1). In our MRM experiment [28], triple quadrupole linear ion trap MS (4000 Qtrap, coupled with a nano Tempo MDLC, Applied Biosystems) was performed; the detailed procedure is previously described [28]. Data were processed using the MultiQuant program (Applied Biosystems, version 1.0), and each peak area of the transitions was normalized to an input internal standard (Q1/Q3 transitions at $542.3 / 636.3 \mathrm{~m} / \mathrm{z}$ for beta-galactosidase peptide) [28]. In the statistical analysis, receiver operating characteristic (ROC) curves and interactive plots were generated using Medicalc (MedCalc Software, Mariakerke, Belgium, version 10.0.1.0).

\section{Results}

3.1. Identification of Urinary Proteomes from Normoalbuminuric and Microalbuminuric Patients. For the iTRAQ experiments, 3 biological replicates (biological replicate 1 was 


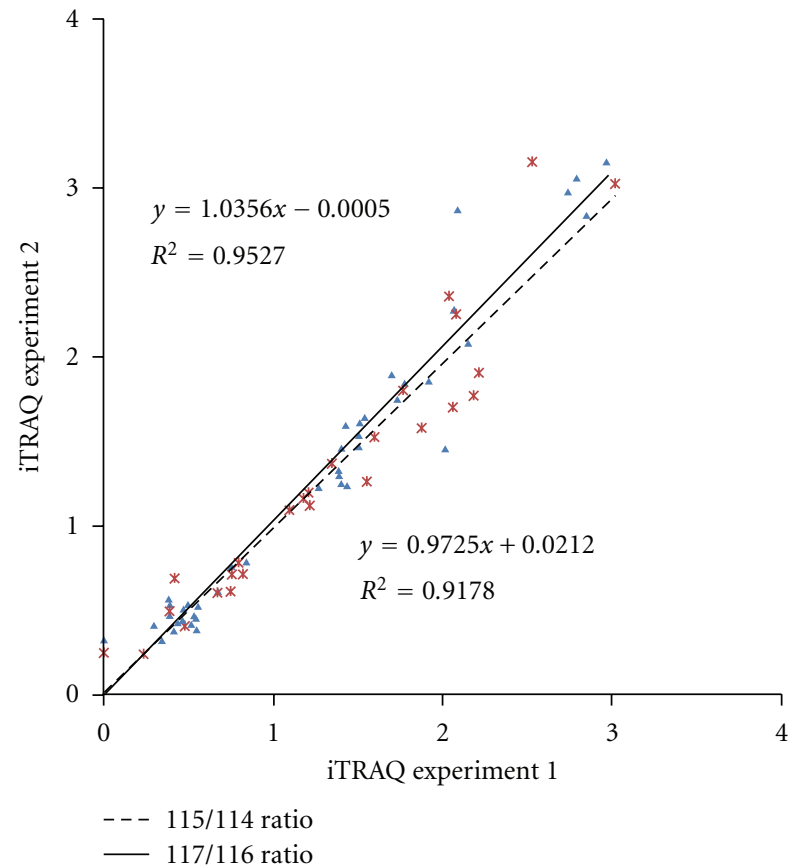

(a)

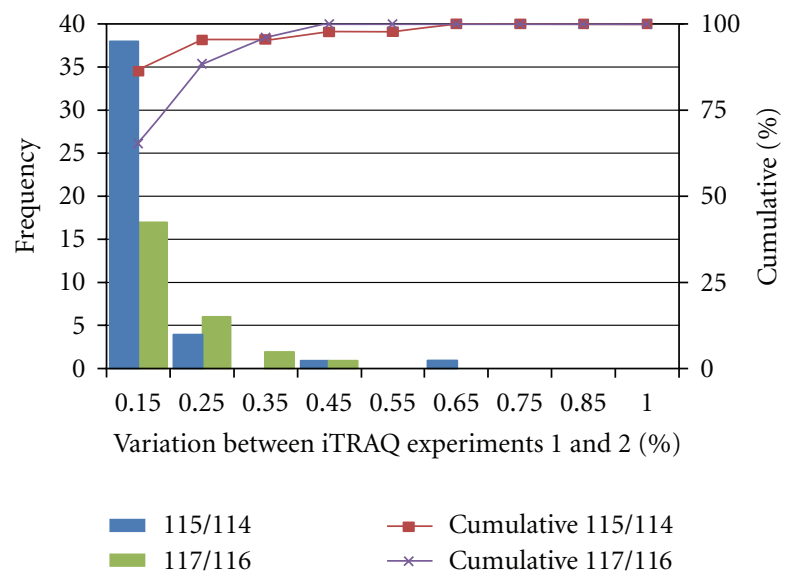

(b)

Figure 2: Correlation between the 2 technical replicates and determination of the cutoff value for significant fold changes. (a) Plots of iTRAQ ratios for two technical replicates. Forty-four proteins were commonly observed from technical replicate 1 (labeled 115/114), and 26 proteins were commonly observed from technical replicate 2 (labeled $117 / 116)$. These differentially excreted proteins $(P$ value $<0.05$, more than two unique peptides: $>95 \%$ ) were plotted in the linear dynamic range. The technical variations yielded a correlation coefficient of $r^{2}$ $=0.9527$ and $r^{2}=0.9178$ between iTRAQ experiments 1 and 2, respectively. (b) The \% variations for the common proteins from the two technical replicates. The 44 and 26 common proteins from the 2 technical replicates were used as inputs to calculate $\%$ variations. The vertical axis represents the number of proteins, and the horizontal axis denotes $\%$ variation. Ninety percent of the proteins fell within $25 \%$ of the respective experimental variation. Thus, we considered a fold-change of $>1.25$ or $<0.80$, a meaningful cutoff that represented actual differences in the iTRAQ experiments.

labeled (a), replicate 2 was labeled (d), and replicate 3 was labeled (e)); 2 technical replicates (technical replicate 1 was labeled (a) and (c), replicate 2 was labeled (b) and (d)) were generated from normoalbuminuric and microalbuminuric urine (Figure 1). The 3 biological replicates were used to profile and quantitate the urinary proteome; the 2 technical replicates were used solely to determine the cutoff for significant fold-changes.

Seven hundred ten proteins were identified from 21,610 peptides of the 3 combined biological replicates at a minimum confidence level of $95 \%$ (unused ProtScore $>1.3$ ). Of the proteins that were identified by iTRAQ, 27\% comprised 1-peptide proteins; $14 \%$ was 2 -peptide proteins; $8 \%$ was 3peptide proteins; $5 \%$ was 4 -peptide proteins; $46 \%$ comprised proteins that had 5 or more peptides. In our iTRAQ experiment, 83 proteins (unused ProtScore $>1.3$ ) were common to all 3 biological replicates at a minimum confidence level of 95\%, using 3 different pooled urine samples.

\subsection{Determination of Cutoff for Significant Fold-Change in} iTRAQ Experiments. To generate the quantitative proteome using iTRAQ labeling, we first determined the labeling efficiency, which exceeded 98\% (data not shown). Next, the cutoff for significant fold-change was determined based on the 2 technical replicates ((a) and (c) of iTRAQ experiment 1 , (b); (d) of iTRAQ experiment 2) (Figure 1). In the 2 replicate experiments, the number of commonly identified proteins was $173(2[115 / 114]$ ratios from technical replicate 1$)$ and 107 (2 [117/116] ratios from technical replicate 2$)$, and the number of selected proteins was 44 (2 [115/114] ratios from technical replicate 1$)$ and 26 (2 [117/116] ratios from technical replicate 2 ), which were chosen based on the following criteria: it contained more than 2 unique peptides (>95\%), and $P$ value $<0.05$ for the $115 / 114$ and $117 / 116$ reporter ions. The 70 proteins were used to monitor technical variations and confirm the threshold for meaningful differences.

The technical variations for the $115 / 114$ and $117 / 116$ reporter ions, calculated using the ratios of the 44 and 26 commonly observed proteins between the 2 technical replicates, were $r^{2}=0.9527$ and $r^{2}=0.9178$, respectively (Figure 2(a)). Accordingly, 90\% of the commonly observed in the technical replicates fell within $25 \%$ of the respective experimental variation (Figure $2(\mathrm{~b})$ ). Therefore, we set fold-change thresholds of $>1.25$ or $<0.80$ to identify true differences between the expression of $115 / 114$ and $116 / 117$ reporter ions, as described in [23].

3.3. Differential Proteomes between Microalbuminuria and Normoalbuminuria. In our iTRAQ study, we obtained diverse biomarker candidates from 3 pooled biological NA/MA 


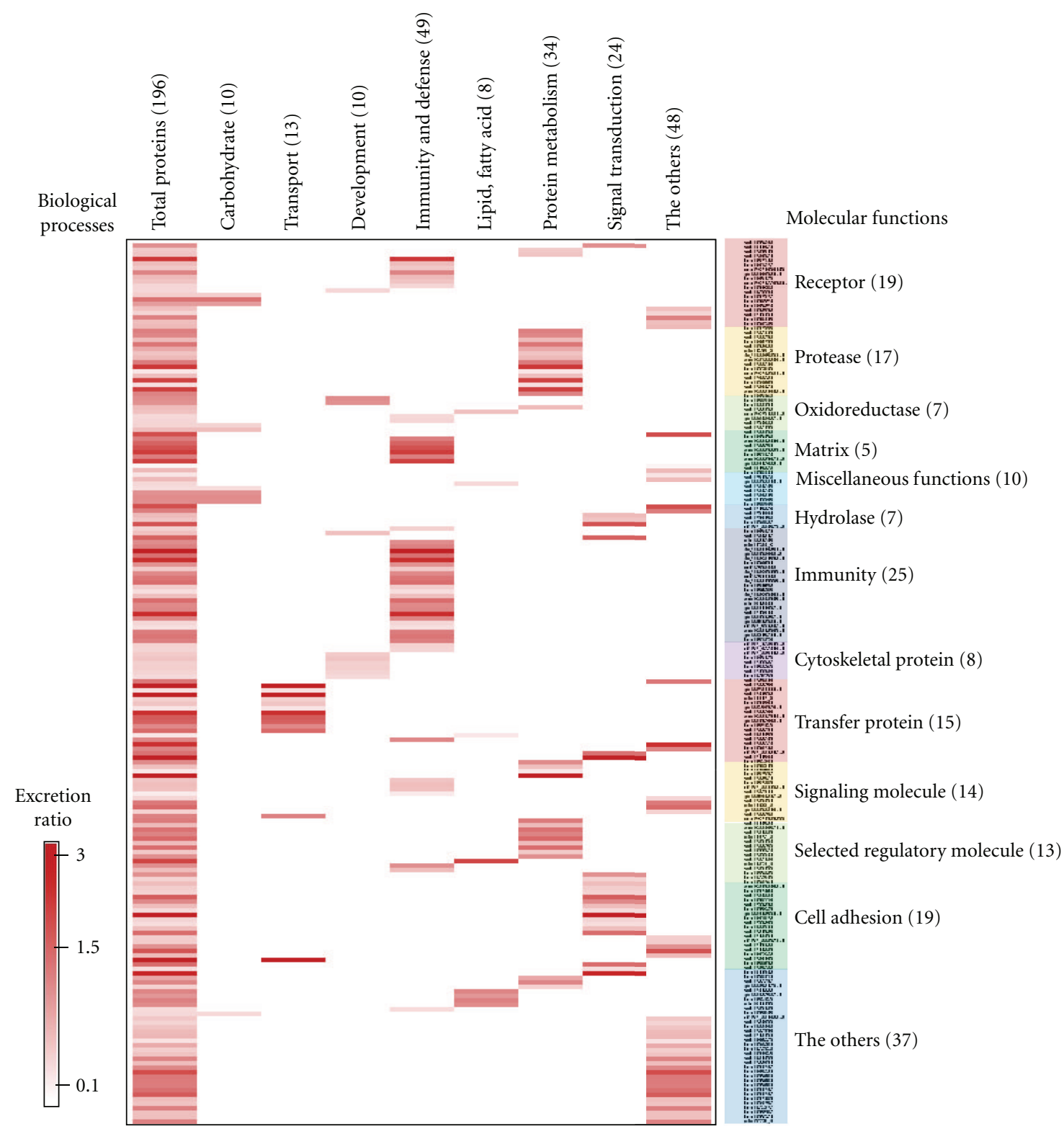

Figure 3: Comprehensive functional annotation of the differentially excreted proteome. The 196 quantitated urinary proteins were annotated for the "biological process" ( $x$-axis) and "molecular function" subcategories ( $y$-axis) in a heatmap. The "biological process" and "molecular function" categories comprised 8 and 13 subcategories, respectively. The 196 quantitated urinary proteins are individually assigned into the "biological process" and "molecular function" subcategories.

urine samples (each pooled NA or MA urine sample consisted of 9 individual urine specimens; thus, the 3 pooled NA, and 3 pooled MA urine samples comprised 54 different individual urine samples). Further, biomarker candidates were confirmed and validated by 2-DE, Western blot, and MRM.

To analyze urinary proteomes in normoalbuminuria and microalbuminuria subjects, 3 biological replicates were generated, wherein 196 proteins met the following criteria: $P$ value $<0.05, \mathrm{EF}<2$, more than 2 unique peptides with $>95 \%$ confidence level, and protein expression $>1.25$ or $<0.80$ for all reporter ions; 99 and 97 proteins were upregulated and downregulated, respectively (Appendix A).

These proteins were further analyzed by differential proteomic expression. All quantified proteins were classified into "biological process" and "molecular function" subcategories using the PANTHER classification program, allowing us to analyze phenotypic features and molecular functions between microalbuminuria and normoalbuminuria (Figure 3). Moreover, to visualize the comprehensive functional annotations graphically, such as in a heatmap, the 196 proteins 


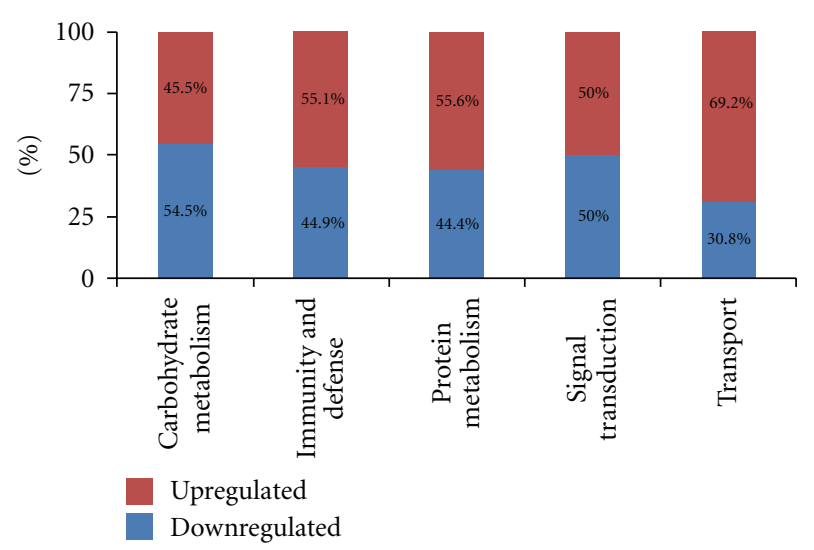

(a)

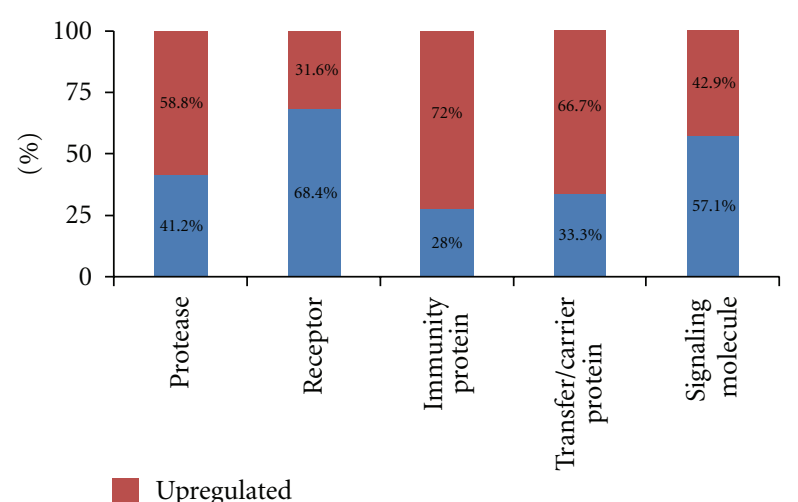

(b)

FIGURE 4: Functional distribution of differentially excreted proteins in microalbuminuria versus normoalbuminuria. Functional classification of differentially excreted proteins into (a) "biological process" and (b) "molecular function." Only 5 major subcategories for "biological process" and "molecular function" are shown; each subcategory is presented as the percentage of up- and down-regulated proteins.

were first categorized by "biological process," and a second dimension was added to coordinate the "molecular function" subcategories (Figure 3). The 196 proteins constituted a preliminary list of biomarkers; Figure 3 shows the expression ratios of the iTRAQ dataset and differentially excreted proteins in microalbuminuria versus normoalbuminuria urine.

\subsection{Classification of Urinary Proteomes in Microalbuminuria} versus Normoalbuminuria. The 196 proteins from the 3 biological replicates were categorized by PANTHER ID number into "biological process" and "molecular function" groups; certain subcategories are summarized in Figures 4(a) and 4(b).

The "biological process" subcategories accounted for 196 differentially excreted proteins, wherein "immunity and defense" and "protein metabolism" represented 49 and 34 of the quantitated proteins, respectively-the 2 largest components (Figure 3). Moreover, in the "biological process" subcategories, "carbohydrate metabolism" (54.5\%) and "signal transduction" (50\%) were downregulated in microalbuminuric versus normoalbuminuric urine (Figure 4(a)). In contrast, $69.2 \%, 55.6 \%$, and $55.1 \%$ of the 196 proteins were upregulated in the "transport," "protein metabolism," and "immunity and defense" subcategories, respectively (Figure 4(a)).

The "molecular function" subcategories accounted for 196 differentially excreted proteins, in which "immunity" and "receptor" represented 25 and 19 of the quantitated proteins, respectively-the 2 largest components (Figure 3). In the "molecular function" subcategories, "receptor" (68.4\%) and "signaling molecule" (57.1\%) proteins were down-regulated in microalbuminuric versus normoalbuminuric urine (Figure $4(\mathrm{~b})$ ). In contrast, $72.0 \%, 58.8 \%$, and $66.7 \%$ of the 196 proteins were up-regulated in the "immunity protein," "protease," and "transfer/carrier protein" subcategories, respectively (Figure 4(b)).

3.5. Differentially Excreted Urinary Proteins Are Associated with Pathogenic Status. One hundred ninety-six tentative biomarker candidates were differentially expressed, based on the iTRAQ data, and they were characterized biologically according to "biological process" and "molecular function." Moreover, in a detailed association study of diabetic nephropathy and differentially excreted proteins using references and databases, we prioritized 10 candidates, of the 196 differentially excreted proteins (Figure 3 ), that were associated with pathogenic status, such as glomerular and tubular dysfunction and other types of diseases. Accordingly, these proteins were classified into categories of pathogenesis in Table 2.

In the 3 biological replicates, transferrin $(T F)$, ceruloplasmin precursor $(C P)$, mannose-binding lectin-associated serine protease-2 precursor (MASP2), alpha-1-antitrypsin $(A 1 A T)$, haptoglobin $(H P)$, and basement membrane-specific heparin sulfate proteoglycan core protein $(H S P G)$ were associated with glomerular dysfunction; except for MASP2 and $H S P G$, all were upregulated in microalbuminuric versus normoalbuminuric urinary proteomes (Table 2).

Moreover, several differentially excreted proteins that were related to tubular dysfunction, such as vitamin D-binding protein $(V D B P)$ and alpha-1-acid glycoprotein 1 precursor $(A G P 1)$ were selected for further validation. VDBP and $A G P 1$ were upregulated in microalbuminuria versus normoalbuminuria (Table 2).

In addition, FABP (fatty acid-binding protein) and PSCA (prostate stem cell antigen) correlate with other types of disease, and PSCA was selected for further validation. In this iTRAQ experiment, $F A B P$ was downregulated, whereas PSCA expression increased in the microalbuminuric versus normoalbuminuric urinary proteome (Table 2 ).

3.6. Identification of Differentially Excreted Proteins Using 2-D $\mathrm{Gel}$ Electrophoresis. Differential protein expression between microalbuminuric and normoalbuminuric urine was also measured using the 2-D gel electrophoresis in pooled NA4 and MA4 urine (Figure 1 and Table 1). In triplicate 2-DE analysis (Figures 5(a) and 5(b)), two proteins (regulator of telomere elongation helicase 1: RTEL1 and serum albumin: 
TABLE 2: Selected 10 differentially excreted proteins related to pathogenic status in microalbumiuric versus normoalbuminuric urines.

\begin{tabular}{|c|c|c|c|c|c|c|c|}
\hline \multirow{2}{*}{ Pathogenic status } & \multirow{2}{*}{$N$} & \multirow{2}{*}{ Number of unique peptides ${ }^{\mathrm{a}}$} & \multirow{2}{*}{ Accession number ${ }^{\mathrm{b}}$} & \multirow{2}{*}{ Gene name $e^{\mathrm{c}}$} & \multicolumn{3}{|c|}{ MA : NA expression } \\
\hline & & & & & iTRAQ $^{\mathrm{d}}$ & $2-\mathrm{DE}^{\mathrm{e}}$ & $\mathrm{WB}^{\mathrm{f}}$ \\
\hline \multirow{6}{*}{ Glomerular dysfunction } & 1 & 15 & $\mathrm{spt} \mid \mathrm{P} 02787$ & $T F$ & 1.86 & - & $4.66 \pm 1.41$ \\
\hline & 2 & 47 & Spt|P00450 & $C P$ & 2.09 & - & $11.16 \pm 0.38$ \\
\hline & 3 & 209 & spt|P01009 & $A 1 A T$ & 1.42 & - & $3.36 \pm 0.03$ \\
\hline & 4 & 49 & spt|P00738 & $H P$ & 2.35 & - & $7.28 \pm 5.52$ \\
\hline & 5 & 10 & $\operatorname{trm} \mid$ Q9UMV3 & MASP2 & 0.29 & $0.10 \pm 0.005$ & - \\
\hline & 6 & 235 & spt|P98160 & $H S P G$ & 0.68 & $0.28 \pm 0.041$ & - \\
\hline \multirow{2}{*}{ Tubular dysfunction } & 7 & 9 & spt|P02774 & $G C, V D B P$ & 2.44 & - & $2.88 \pm 0.11$ \\
\hline & 8 & 414 & spt|P02763 & ORM1, AGP1 & 2.04 & - & $1.82 \pm 0.08$ \\
\hline \multirow{2}{*}{ Other types of diseases } & 9 & 9 & spt|Q01469 & $F A B P$ & 0.29 & $0.27 \pm 0.037$ & - \\
\hline & 10 & 5 & $\operatorname{trm} \mid \mathrm{O} 43653$ & PSCA & 1.70 & - & - \\
\hline
\end{tabular}

${ }^{\mathrm{a}}$ The numbers of unique peptides and MS/MS spectrum observed by ProteinPilot software were determined only for those peptides with $\geq 95 \%$ confidence. ${ }^{\mathrm{b}}$ Accession numbers represent entries in the Human CDS database (human KBMS 5.0, 2005-03-02; a total of 187,748 entries provided by Applied Biosystems). ${ }^{\mathrm{c}}$ Gene name from the Expasy database correspond to protein accession number ${ }^{\mathrm{b}}$ from the Human CDS database (human KBMS 5.0, 2005-03-02; a total of 187,748 entries provided by Applied Biosystems). ${ }^{\mathrm{d}-\mathrm{f}}$ Ratio of differentially excreted protein for iTRAQ, 2-DE and WB in microalbumiuric versus normoalbuminuric urines, respectively.

$A L B)$ were upregulated, and 5 proteins (basement membrane-specific heparan sulfate proteoglycan core protein: $H S P G$, fatty acid-binding protein: FABP, mannose binding lectin-associated serine protease-2: MASP2, AMBP protein: $A M B P$, and Fibulin-5: FBLN5) were downregulated in microalbuminuric urine (Figures 5(a) and 5(b)).

Of the 7 proteins that were identified by $2-\mathrm{DE}, 4$ had the same pattern of differential excretion as in the iTRAQ experiment. Specifically, the spots that corresponded to serum albumin were upregulated by 2-DE (Figure 3(b), spots 1: $50.8 \pm 15.3,2: 17.5 \pm 4.0$, and $3: 14.2 \pm 2.5)$ and iTRAQ (iTRAQ: 3.09 ). In contrast, HSPG (spot 4: $0.28 \pm 0.041$ and iTRAQ: 0.68), FABP (spot 5: $0.27 \pm 0.037$ and iTRAQ: 0.29), and MASP2 (spot 6: $0.10 \pm 0.005$ and iTRAQ: 0.29) were significantly downregulated in both techniques (Table 3 ). AMBP (spot 7) was downregulated by 2-DE $(0.20 \pm 0.002)$ but upregulated in the iTRAQ experiment (1.44). Two proteins were identified by $2-\mathrm{DE}$ alone-regulator of telomere elongation helicase 1 (spot 8: $3.0 \pm 0.5$ ) was upregulated and fibulin-5 precursor (spot 9: $0.12 \pm 0.007$ ) was downregulated (Table 3).

3.7. Validation of Differentially Expressed Proteins from iTRAQ by Western Blot. To validate the differentially excreted proteins from the iTRAQ results, 6 proteins (TF, CP, A1AT, $V D B P, A G P 1$, and $H P$ ) that were associated with pathogenic status were subjected to Western blot. The Western blot results were consistent with the iTRAQ findings (Figure 6 and Table 2): TF $(4.66 \pm 1.41$ and $P<0.0005), C P(11.16 \pm$ 0.38 and $P<0.01), A 1 A T(3.36 \pm 0.03$ and $P<0.005), V D B P$ $(2.88 \pm 0.11$ and $P<0.05), A G P 1(1.82 \pm 0.08$ and $P<0.05)$, and $H P(7.28 \pm 5.52$ and $P<0.05)$ were upregulated in microalbumiuric versus normoalbuminuric urine.

3.8. MRM Validation for 7 Selected Biomarker Candidates. To verify the 7 biomarker candidates ( TF, CP, A1AT, HP, $V D B P, A G P 1$, and $P S C A$ ), MRM was performed using 9 individual normoalbuminuric and 14 microalbuminuric samples
(Figure 1 and Table 1). The peak area for each Q1/Q3 transition (Table 4) for the candidates was first normalized to the peak area of beta-galactosidase that was spiked with $50 \mathrm{fmol}$ as the internal standard and compared between microalbuminuric versus normoalbuminuric samples.

MRM validation was assessed by interactive plots and ROC curves, represented by the peak area of each Q1/Q3 transition. Figure 7 shows the interactive plots and ROC curves for TF, CP, A1AT, VDBP, AGP1, HP, and PSCA with regard to sensitivity, specificity, and relative concentrations versus beta-galactosidase. In the ROC curves, TF, A1AT, $A G P 1, H P$, and PSCA had excellent area under the curve (AUC) values $(0.762,0.849,0.873,0.754$, and 0.825 , resp.), as did $C P$ and $V D B P$, to a lesser extent (0.683 and 0.675 , resp.) (Figure 7). Particularly, the merged ROC curve combining 3 biomarker candidates (alpha-1-antitrypsin, alpha-1-acid glycoprotein 1, and prostate stem cell antigen) resulted in the improved AUC value of 0.921 , which is greater than those of the individual proteins $(0.849,0.873$, and 0.825 for alpha-1antitrypsin, alpha-1-acid glycoprotein 1, and prostate stem cell antigen, resp.) (Figure 8).

In the interactive plots, $T F, C P, A 1 A T, V D B P, A G P 1$, and $P S C A$ were upregulated in microalbuminuric versus normoalbuminuric urine, whereas $H P$ was down-regulated. $T F$, $C P, A 1 A T, V D B P, A G P 1$, and PSCA had the same excretion patterns by iTRAQ and western blot; conversely, HP had the opposite excretion pattern.

\section{Discussion}

4.1. Differentially Excreted Proteomes between Microalbuminuria and Normoalbuminuria. To identify and quantify proteins that were associated with diabetic nephropathy in microalbuminuric and normoalbuminuric urine, we used relative quantitative proteomic techniques, such as iTRAQ, 2DE, Western blot, and MRM. In our iTRAQ experiment, 710 urinary proteins were identified at a $>95 \%$ confidence level, of which 196 were differentially excreted by $>1.25$ 
TABLE 3: Differentially expressed proteins by 2-DE in microalbuminuria versus normoalbuminuria.

\begin{tabular}{|c|c|c|c|c|c|c|c|}
\hline Gene name ${ }^{a}$ & $\begin{array}{l}\text { Accession } \\
\text { number }^{\mathrm{b}}\end{array}$ & $\begin{array}{c}\text { Up- } \\
\text { /down- } \\
\text { regulated }\end{array}$ & $\begin{array}{l}\text { Mol. Mass, } \\
\mathrm{Da}(\mathrm{pI})^{\mathrm{c}}\end{array}$ & $\begin{array}{l}\text { Peptides } \\
\text { matched }\end{array}$ & $\begin{array}{l}\text { Total ion } \\
\text { C.I.\% }{ }^{\mathrm{d}}\end{array}$ & $\begin{array}{l}\mathrm{Ma} / \mathrm{Na} \\
(2-\mathrm{DE})^{\mathrm{f}}\end{array}$ & $\begin{array}{c}\mathrm{Ma} / \mathrm{Na} \\
\left(^{(\mathrm{iTRAQ})^{\mathrm{g}}}\right.\end{array}$ \\
\hline$A L B$ & P02768 & Up & $\begin{array}{c}71317.2 \\
(5.92)\end{array}$ & 2 & 100.00 & $50.8 \pm 15.3$ & 3.09 \\
\hline$A L B$ & P02768 & Up & $\begin{array}{c}71317.2 \\
(5.92)\end{array}$ & 2 & 99.84 & $17.5 \pm 4.0$ & 3.09 \\
\hline$A L B$ & P02768 & Up & $\begin{array}{c}71317.2 \\
(5.92)\end{array}$ & 2 & 100.00 & $14.2 \pm 2.5$ & 3.09 \\
\hline$H S P G$ & P98160 & Down & $\begin{array}{c}468527.5 \\
(6.06)\end{array}$ & 2 & 100.00 & $0.28 \pm 0.041$ & 0.68 \\
\hline$F A B P$ & Q01469 & Down & $\begin{array}{c}15496.7 \\
(6.6)\end{array}$ & 1 & 97.76 & $0.27 \pm 0.037$ & 0.29 \\
\hline$M A S P 2$ & Q9UMV3 & Down & $\begin{array}{c}75684.6 \\
(5.47)\end{array}$ & 2 & 100.00 & $0.10 \pm 0.005$ & 0.29 \\
\hline$A M B P$ & P02760 & Down & $\begin{array}{l}38974 \\
(5.95)\end{array}$ & 2 & 99.91 & $0.20 \pm 0.002$ & 1.44 \\
\hline RTEL1 & Q9NZ71 & Up & $\begin{array}{c}152278.2 \\
(8.68)\end{array}$ & 1 & 96.77 & $3.0 \pm 0.5$ & - \\
\hline FBLN5 & Q9UBX5 & Down & $\begin{array}{c}50146.7 \\
(4.58) \\
\end{array}$ & 1 & 99.36 & $0.12 \pm 0.007$ & - \\
\hline
\end{tabular}

${ }^{\mathrm{a}-\mathrm{b}}$ Gene name from the Expasy database correspond to protein accession number. ${ }^{\mathrm{b}}$ Accession numbers represent entries in the Human CDS database. ${ }^{\mathrm{c}}$ Molecular mass (mol. mass) is presented by Da, while isoelectric point stands for pI. ${ }^{\mathrm{d}}$ Total ion score and total ion CI \% for MALDI-TOF/TOF were calculated using GPS v3.5 in the MASCOT search program (v2.0). ${ }^{\mathrm{f}-\mathrm{g}}$ Ratio of differentially excreted protein for 2-DE and iTRAQ in microalbumiuric versus normoalbuminuric urines, respectively. Data are expressed as the mean \pm SD.

TABLE 4: Parameters of MRM Experiment for seven candidate proteins.

\begin{tabular}{|c|c|c|c|c|c|c|}
\hline Protein name & $\mathrm{Q} 1^{\mathrm{a}}$ & $\mathrm{Q}^{\mathrm{b}}$ & Sequence ${ }^{c}$ & Fragment $^{\mathrm{d}}$ & Charge $^{\mathrm{e}}$ & $C E^{f}$ \\
\hline \multirow{3}{*}{ Transferrin } & 482.8 & 682.4 & APNHAVVTR & y6 & $2+$ & 26 \\
\hline & 315.2 & 558.3 & AVGNLR & y5 & $2+$ & 19 \\
\hline & 315.2 & 459.3 & AVGNLR & $\mathrm{y} 4$ & $2+$ & 19 \\
\hline \multirow{2}{*}{ Ceruloplasmin } & 686.4 & 1080.0 & GAYPLSIEPIGVR & y10 & $2+$ & 35 \\
\hline & 686.39 & 870.5 & GAYPLSIEPIGVR & y8 & $2+$ & 35 \\
\hline \multirow{4}{*}{ Alpha-1-antitrypsin } & 555.81 & 997.5 & LSITGTYDLK & y9 & $2+$ & 29 \\
\hline & 555.81 & 797.4 & LSITGTYDLK & y7 & $2+$ & 29 \\
\hline & 393.2 & 587.3 & VVNPTQK & y5 & $2+$ & 22 \\
\hline & 393.2 & 473.3 & VVNPTQK & y5 & $2+$ & 22 \\
\hline \multirow{2}{*}{ Haptoglobin precursor } & 729.8 & 1084.5 & NLFLNHSENATAK & y10 & $2+$ & 37 \\
\hline & 352.2 & 517.3 & VSVNER & $\mathrm{y} 4$ & $2+$ & 20 \\
\hline \multirow{2}{*}{ Vitamin D-binding protein } & 400.2 & 700.4 & VLEPTLK & y6 & $2+$ & 26 \\
\hline & 400.2 & 587.3 & VLEPTLK & y5 & $2+$ & 26 \\
\hline \multirow{3}{*}{ Alpha-1-acid glycoprotein 1} & 556.8 & 811.4 & SDVVYTDWK & y6 & $2+$ & 29 \\
\hline & 580.8 & 974.5 & WFYIASAFR & y8 & $2+$ & 31 \\
\hline & 580.8 & 827.4 & WFYIASAFR & y7 & $2+$ & 31 \\
\hline \multirow{2}{*}{ Prostate stem cell antigen } & 501.0 & 830.5 & AVGLLTVISK & y8 & $2+$ & 30 \\
\hline & 501.0 & 660.4 & AVGLLTVISK & y6 & $2+$ & 30 \\
\hline
\end{tabular}

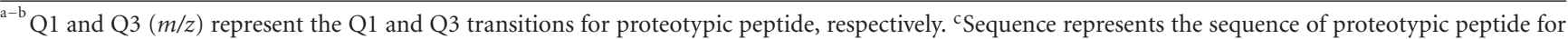
target protein. ${ }^{\mathrm{d}}$ Fragment type indicates the ion type of the Q3 transition. ${ }^{\mathrm{e}} \mathrm{Charge}$ represents the charge state of precursor ion. ${ }^{\mathrm{f}} \mathrm{CE}$ represents collision energy.

or $<0.80 \_99$ and 97 proteins were up- and down-regulated, respectively (Appendix A).

Recently, the Urinary Protein Biomarker (UPB) database was constructed and published, in which 205 publications were curated manually [29]. Using this database, we can easily determine whether a biomarker candidate has been identified by another group for the same disease and evaluate its disease specificity. Thirty-six of the 196 quantified proteins from our ITRAQ experiment were registered in the UPB database; the remaining 160 proteins were not listed. 
pI

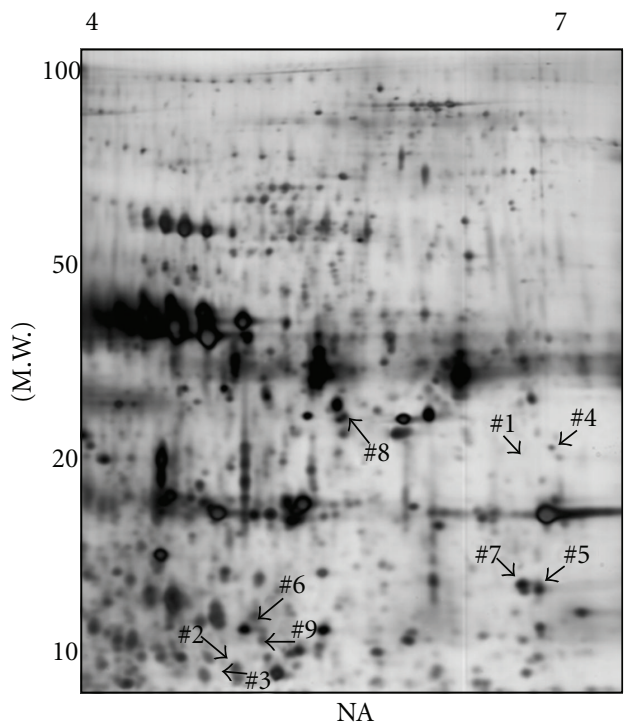

pI

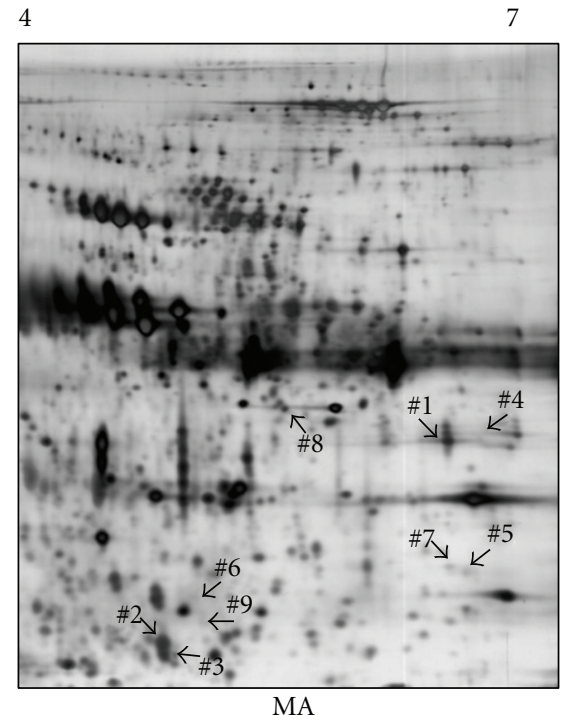

(a)
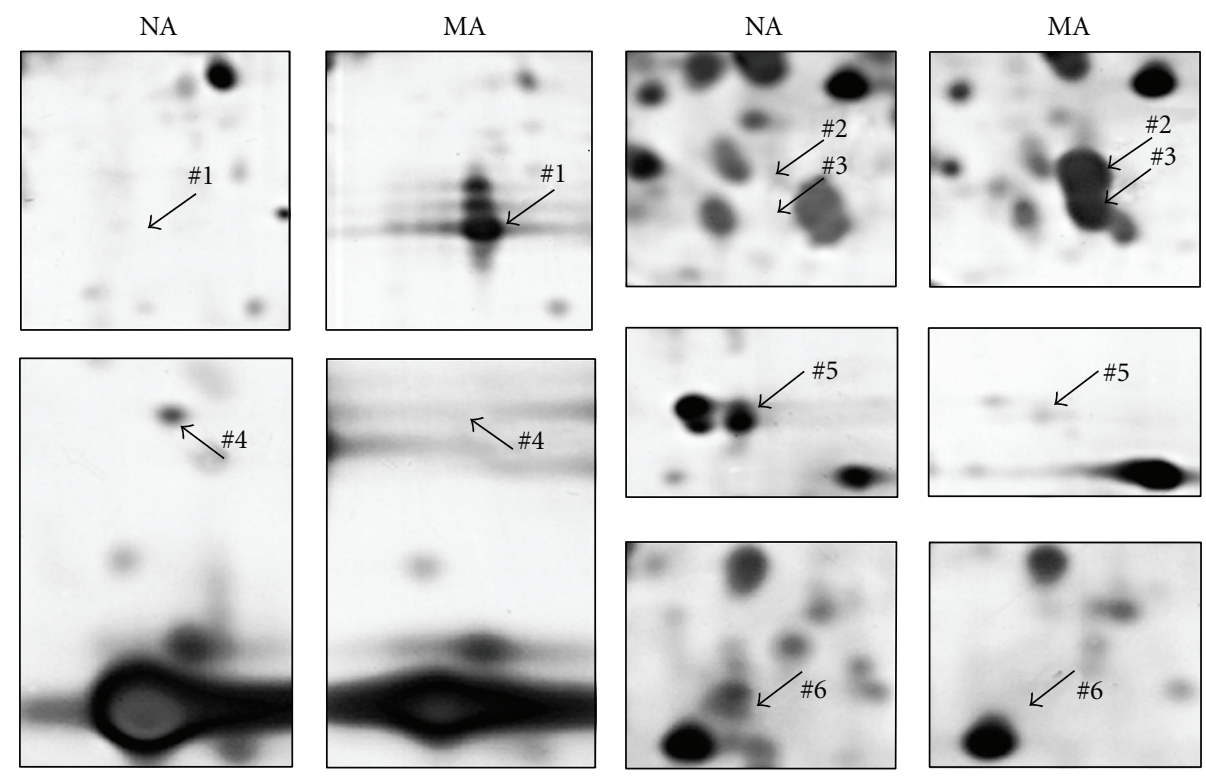

(b)

FIGURE 5: Differentially excreted proteins by 2-DE in microalbuminuria versus normoalbuminuria. (a) Representative whole 2-DE images of normoalbuminuric (NA) and microalbuminuric (MA) urine. Total protein (100 $\mu \mathrm{g})$ samples were loaded onto IPG strips (pH 4-7, nonlinear) for IEF and separated in the second dimension on a $12 \%$ polyacrylamide gel. The horizontal and vertical axes represent $\mathrm{p} I$ and molecular weight, respectively. The arrowed numbers denote for differentially excreted proteins and correspond to the proteins in Table 3 . (b) Magnified sections of differentially excreted proteins in the 2-DE gel. Some arrowed proteins in the 2-DE gel (Figure 5(a)) were magnified side by side to compare their relative expression.

Subsequently, 196 differentially excreted proteins yielded 10 preliminary biomarker candidates for further validation.

The "molecular function" subcategories accounted for 196 differentially excreted proteins, of which "immunity" represented 25 of the quantitated proteins - the largest component (Figure 3). This proportion reflects the increased inflammatory reactions and higher vascular lesion counts in kidneys during the development of diabetic nephropathy.
Through detailed association searches between diabetic nephropathy and the 196 differentially excreted proteins using relevant databases and references, we identified and classified several biomarker candidates that were associated with pathogenic status, such as glomerular and tubular dysfunction and other types of disease (Table 2).

Consequently, 10 proteins were selected for preliminary validation studies such as $2-\mathrm{DE}$, Western blot, and MRM. 


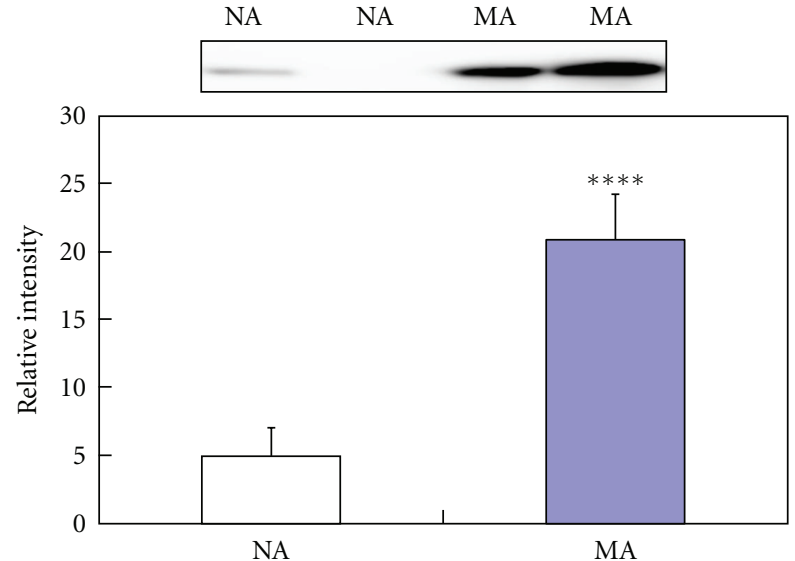

(a)

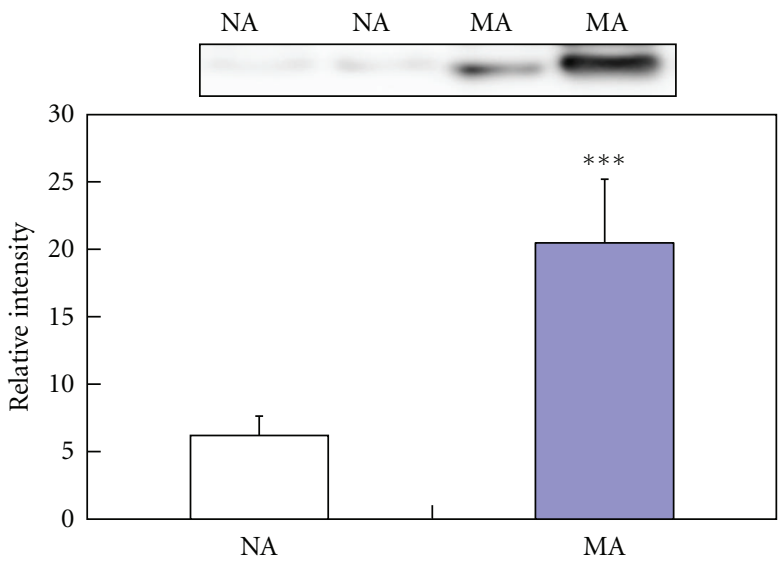

(c)

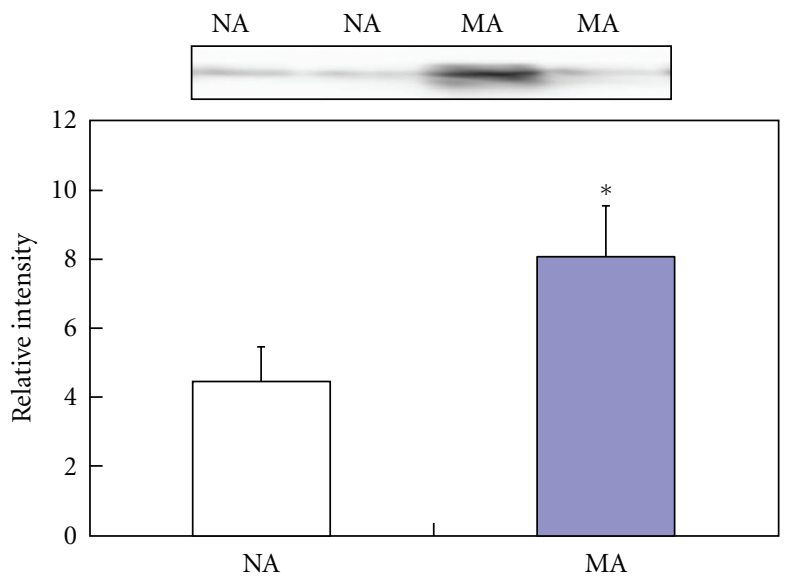

(e)

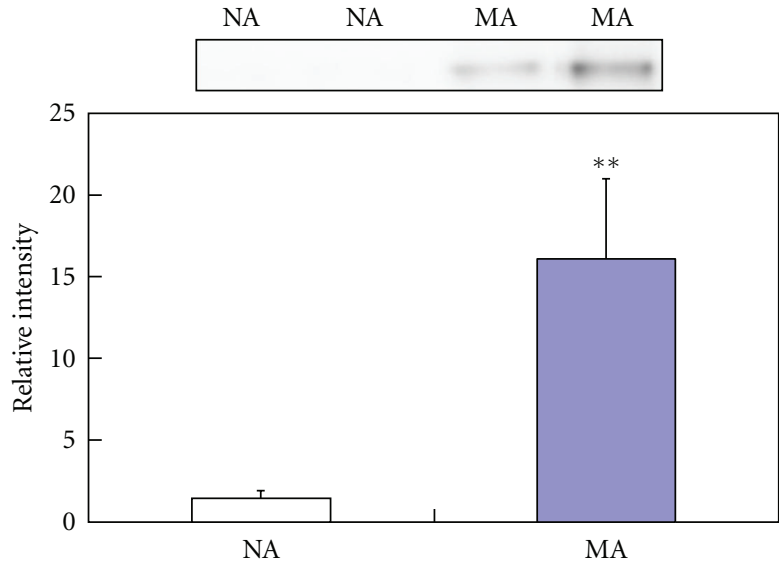

(b)

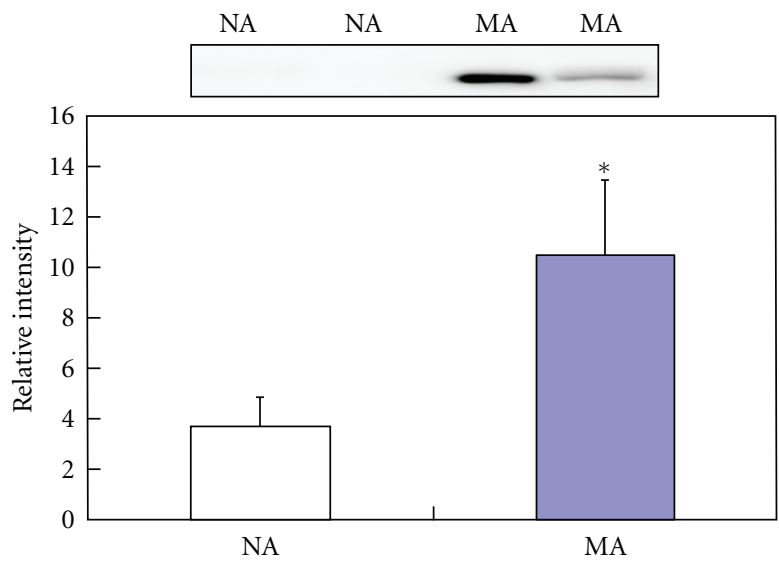

(d)

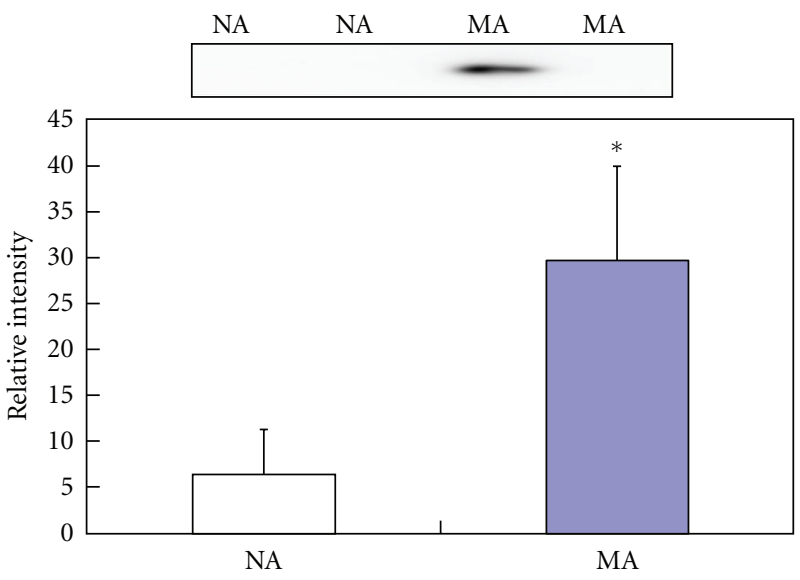

(f)

FIGURE 6: Validation using Western blot for six representative differentially excreted proteins. The concentrations of transferrin (a), ceruloplasmin (b), $\alpha 1$-antitrypsin (c), vitamin D-binding protein (d), $\alpha 1$-acid glycoprotein (e), and haptoglobin (f) are significantly higher in microalbuminuric patients versus normoalbuminuric urine. The relative intensities on the vertical axis indicate normalized values versus the representative control. Each bar represents the mean \pm SEM, based on the relative intensities of the gel bands. Statistical significance for the differences $\left({ }^{*} P<0.05\right.$; ${ }^{* *} P<0.01$; ${ }^{* *} P<0.005$; $\left.{ }^{* * *} P<0.0005\right)$ were determined by paired Student's $t$-test. Two representative NA and MA blots are shown at the top of the bar graphs. 

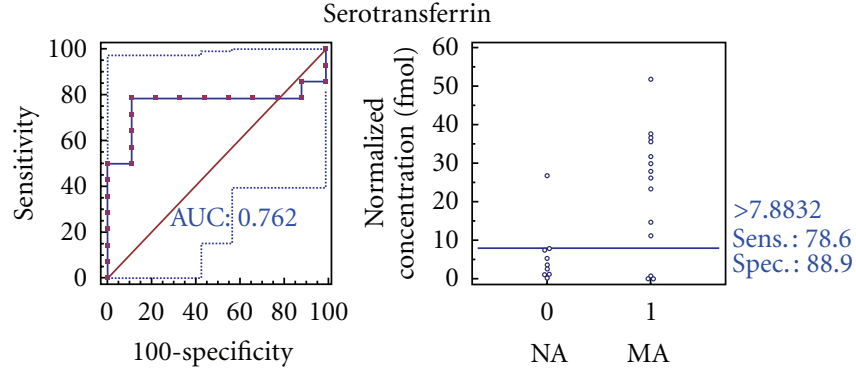

(a)

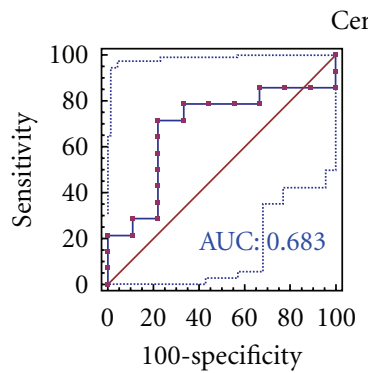

Ceruloplasmin

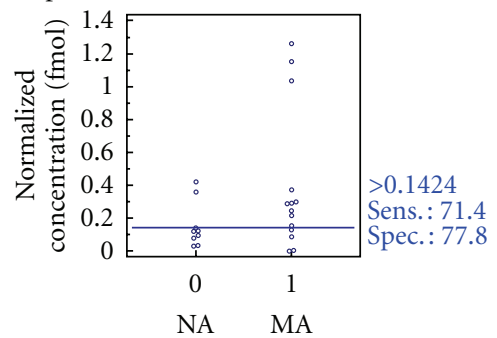

(b)
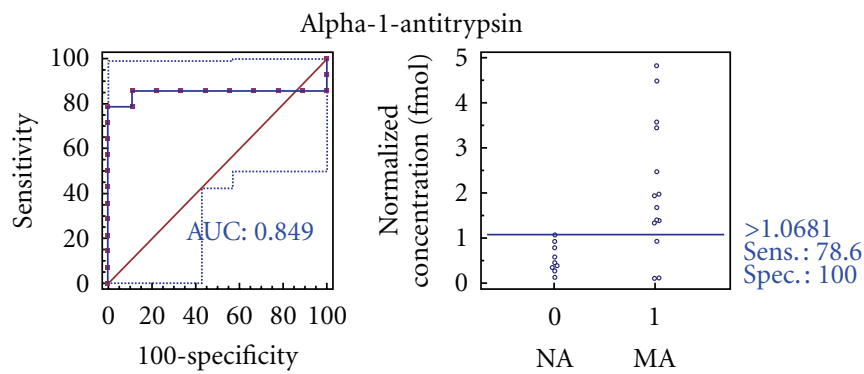

(c)
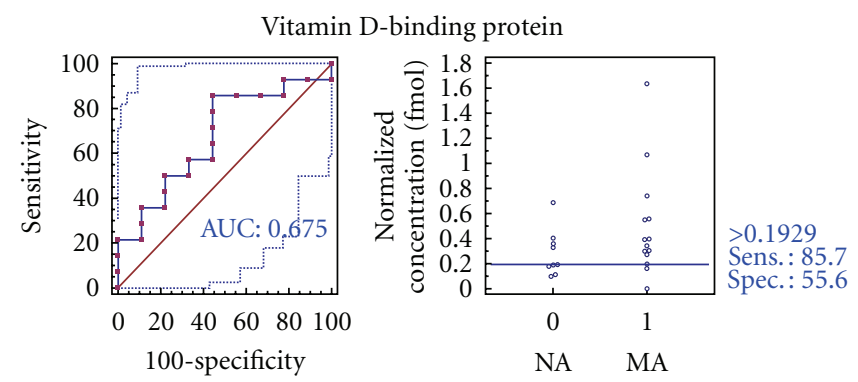

(d)
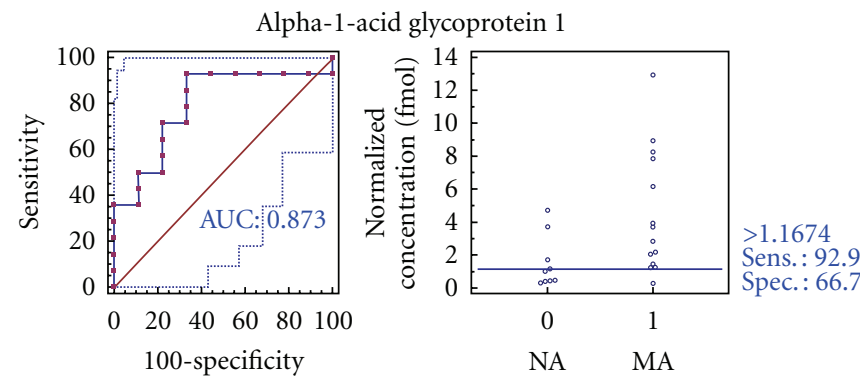

(e)

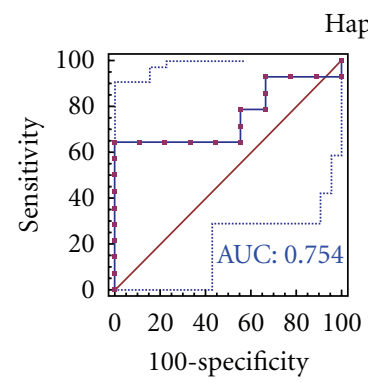

aptoglobin

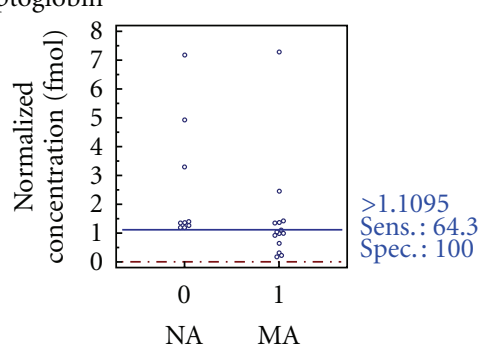

(f)
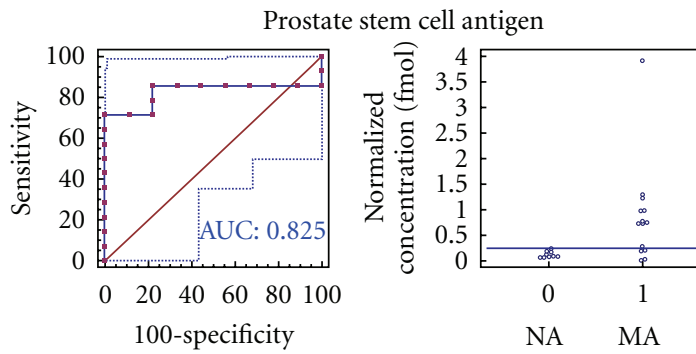

(g)

Figure 7: ROC curves and interactive plots for MRM validation in normoalbuminuric versus microalbuminuric urine. Seven biomarker candidates (TF, CP, A1AT, VDBP, AGP1, HP, and PSCA) were validated by MRM, in which 9 normoalbuminuric and 14 microalbuminuric urine samples were used. (a)-(g) Interactive plots and ROC curves for TF, $C P, A 1 A T, V D B P, A G P 1, H P$, and PSCA. In the ROC curves, the solid lines represent the corresponding score in sensitivity ( $x$-axis) and 100 -specificity ( $y$-axis). In the interactive plots, the $y$-axis indicates the normalized concentration of the target protein against the spiked internal standard ( $50 \mathrm{fmol}$ of beta-galactosidase peptide). Sens. and Spec. represent the sensitivity and specificity for the target proteins, respectively. The AUC values are shown inside the ROC curves.

For example, 3 proteins (HSPG, FABP, and MASP2) that were identified by 2-DE had the same pattern of differential excretion in the 2-DE and iTRAQ experiments (Table 2). Six differentially excreted proteins ( $T F, C P, A 1 A T, V D B P, A G P 1$, and $H P$ ) were validated by Western blot, for which the patterns of excretion were consistent with the iTRAQ results (Table 2).
Recently, an optimized quantitative proteomic strategy in urinary proteomic analysis was proposed for urine biomarker discovery using a small set of samples [21]. According to this report, the initial amount of proteins that is analyzed and the precipitation method (methanol precipitation) of the urine proteins are critical. Notably, our preparation methods for urinary proteomes approximated this optimized 


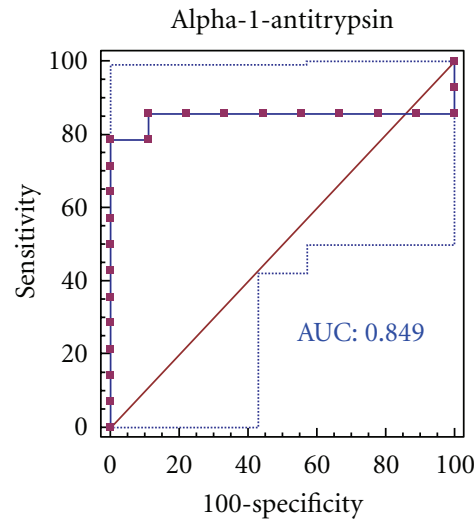

(a)

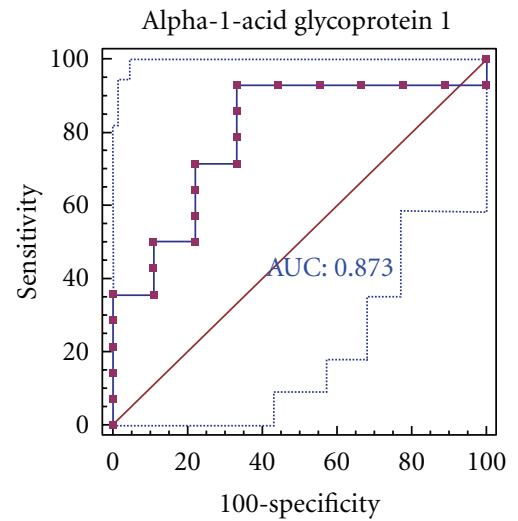

(b)

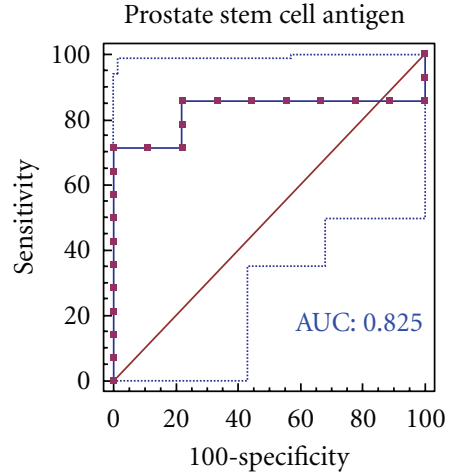

(c)

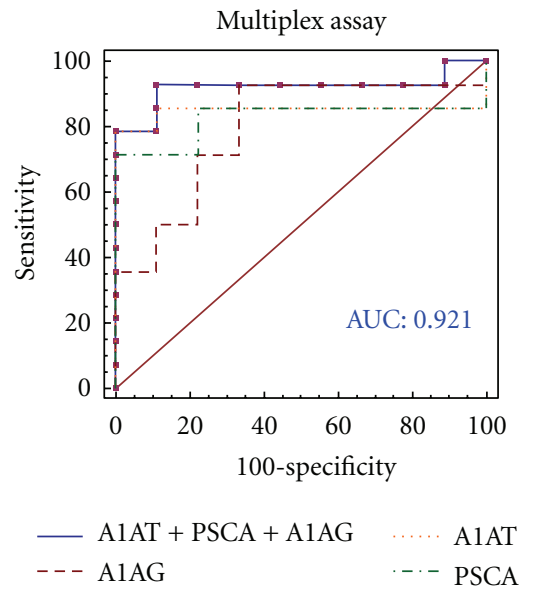

(d)

FIGURE 8: ROC curves for three candidate biomarkers and the 3-marker panel. MRM validation was performed for (a) alpha-1-antitrypsin, (b) alpha-1-acid glycoprotein 1, (c) prostate stem cell antigen, and (d) their combination, generating AUC values of 0.849, 0.873, and 0.825, respectively, whereas the combination resulted in a merged AUC of 0.921.

protocol, although we used acetone precipitation instead of methanol precipitation.

Nevertheless, an advantage of our study was that we used a large collection of urine samples from 86 diabetic patients to perform 3 iTRAQ experiments, including 3 biological replicates and 2 technical replicates, resulting in more reliable statistical significance.

4.2. Differentially Excreted Proteome and Glomerular Dysfunction. Glomerular dysfunction is caused by GBM thickening and mesangial expansion due to ECM accumulation [2], and several proteins, such as TF, CP, MASP2, A1AT, HP, and $H S P G$, were associated with glomerular dysfunction.

Transferrin-to-creatinine and ceruloplasmin-to-creatinine ratios are known to reflect changes in renal hemodynamics, and these ratios are significantly higher microalbuminuric patient than normoalbuminuric patients [30]. This result is caused by elevated intraglomerular hydraulic pressure, which leads to the development of diabetic glomerulosclerosis [30-32]. Moreover, $T F$ and $C P$ were listed in the UPB database, showing upregulation. TF is associated with diabetic nephropathy, normoalbuminuric type 2 diabetes, kidney calculi, and ureteropelvic junction obstruction, whereas $C P$ is linked to diabetic nephropathy and normoalbuminuric type 2 diabetes. In our iTRAQ and 2-DE, $T F$ and $C P$ urinary proteins were commonly upregulated in the comparison of microalbuminuric and normoalbuminuric urinary proteome (Table 2 ).

MASP2 is a serum protease that activates the complement cascade, which regulates the maintenance of glomerular permeability and the pathogenesis of focal segmental glomerulosclerosis [33]. MASP2 was not listed in the UPB database, and in this iTRAQ and 2-DE, this protein was commonly downregulated in comparison of microalbuminuric and normoalbuminuric urinary proteome (Table 2 ).

A1AT is a serine protease inhibitor, which prevents neutrophil elastase by degrading ECM proteins, which maintains vascular elasticity and glomerular integrity $[13,34]$. In a previous study, A1AT excretion was elevated in microalbuminuria, which can cause matrix molecules to accumulate [35]. A1AT was also listed in the UPB database, showing upregulation; it is associated with diabetic nephropathy, severe acute pancreatitis, kidney calculi, nephrotic syndrome, and ureteropelvic junction obstruction. In our iTRAQ and 
Serotransferrin (transition 1)

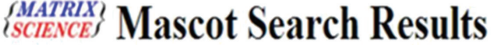

Peptide View

MS/MS Fragmentation of APNHAVVTR

Found in TRFE HUMAN, Serotransferrin OS=Homo sapiens GN=TF PE=1 SV=2

Click mosse within plot rea to 2000 in by factor of tro a bout that point

Or, Plotion 150 to 800 Da Fulramg

Label all possible matches 0 Label matcies ssed for scoring $\odot$

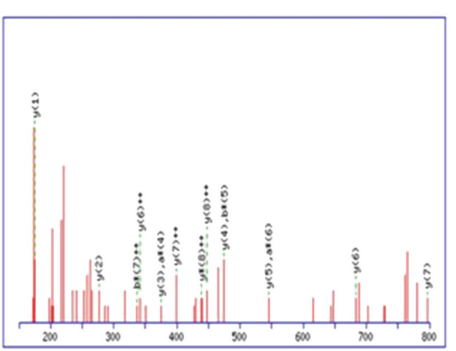

\begin{tabular}{|c|c|c|c|c|c|c|c|c|c|c|c|c|}
\hline ; 1 & $i$ & & $r^{+}$ & $b$ & $r$ & b & $r^{*-1}$ & 54 & ! & $\vec{r}$ & $!$ & $r^{+*}$ \\
\hline 1 40,45 & $25: 24$ & & & $7204+4$ & 3655 & & & 1 & & & & 9 \\
\hline 2141002 & 70648 & & & 16900"? & $8505 \%$ & & & \multicolumn{5}{|c|}{ 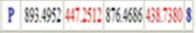 } \\
\hline $33515: 1$. & 180062 & $38: 118$ & 119565 & $83: 190$ & 14007 & & 5133504 & \multicolumn{5}{|c|}{ 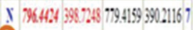 } \\
\hline 439220411 & 1866657 & 356.17 & $1800 \% 4$ & 1001980 & 201060: & & 400009 & \multicolumn{5}{|c|}{ 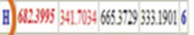 } \\
\hline 54632412.2 & $3312 \%$ & H.521: & 236110 & 191.336 & $2.61212 !$ & & 6356604 & \multicolumn{5}{|c|}{ 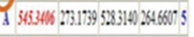 } \\
\hline 65633985: & 286584 & & 7.1.15\% & 19003045 & & & 0.281 .166 & \multicolumn{5}{|c|}{ 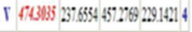 } \\
\hline $76613703:$ & $33119 \%$ & 6435: & $3269 \%$ & 469395 & $35519 \%$ & & 4366668 & \multicolumn{5}{|c|}{ 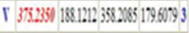 } \\
\hline 87644573 & 361765 & & 373013: & 290.406 & ;is: & & 1361300 & \multicolumn{5}{|c|}{ 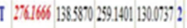 } \\
\hline & & & & & & & & & 33114 & $8866 ;$ & 1569 & 994901 \\
\hline
\end{tabular}

ROC curve

Serotransferrin APNHAVVTR_2+/y6 (482.8/682.4)
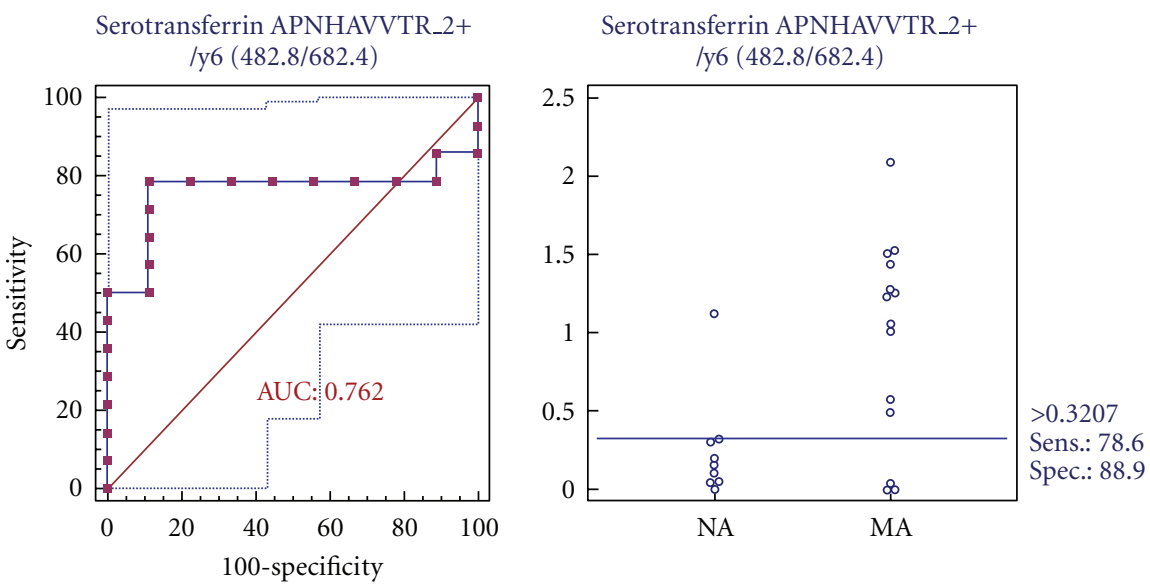

Figure 9

Western blot, A1AT was also upregulated in the comparison of microalbuminuric and normoalbuminuric urinary proteome (Table 2).

Haptoglobin $(H P)$ is an acute phase protein that binds to free hemoglobin $(\mathrm{Hb})$ with the highest affinity. Formation of the Hb-HP complex prevents the loss of renal iron and oxidative damage that are driven by free $\mathrm{Hb}$. Specifically, $H P$ mediates complement-dependent podocyte damage [36]. Thus, complement activation results in the release of proteases, oxidants, and growth factors, damaging the functional integrity of the GBM. HP, which was downregulated and is associated with diabetic nephropathy and type 2 diabetes mellitus, appeared in the UPB database. However, in our ITRAQ and Western blot, $H P$ was commonly upregulated in the comparison of microalbuminuric and normoalbuminuric urinary proteome (Table 2).

HSPG is present in the basement membrane of every vascularized organ, including the GBM. The highly negatively charged side chains of HSPG are important determinants for the charge-selective permeability of the GBM [37]. Under hyperglycemic conditions, the loss of HSPG from the GBM alters the charge-selective properties of the glomerular capillary, causing increased filtration of negatively charged albumin $[37,38]$. HSPG is not registered in the UPB database, and in our ITRAQ and 2-DE, HSPG was commonly downregulated in the comparison of microalbuminuric and normoalbuminuric urinary proteome (Table 2).

\subsection{Differentially Excreted Proteome and Tubular Dysfunction} and Other Types of Diseases. Low-molecular-weight proteins, such as $V D B P$ and $A G P 1$, are associated with kidney tubular dysfunction and were commonly upregulated in the iTRAQ and Western blot (Table 2). Unlike high-molecular-weight proteins, they are filtered in the glomerulus on the basis of charge selectivity and pore size of the GBM and are reabsorbed into proximal renal tubules under normal conditions 
Serotransferrin (transition 2-3)

\section{$\{$ SCIENCEX\} $\}$ Mascot Search Results}

Peptide View

MS/MS Fragmentation of AVGNLR

Found in TRFE_HUMAN, Serotransferrin OS=Homo sapiens GN=TF PE=1 SV=2

Click mosses within plot area to $200 \mathrm{~m}$ in by factor of trio about that point

Or, Pottrom 160 to 360 Da fullarge

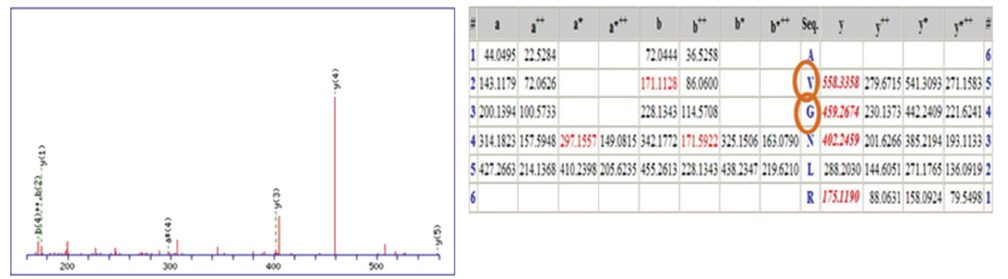

(a)

ROC curve

Serotransferrin_AVGNLR_2+/y5 (315.2/558.3)

Serotransferrin_AVGNLR_2+ /y5 (315.2/558.3)

Serotransferrin_AVGNLR_2+ /y5 (315.2/558.3)
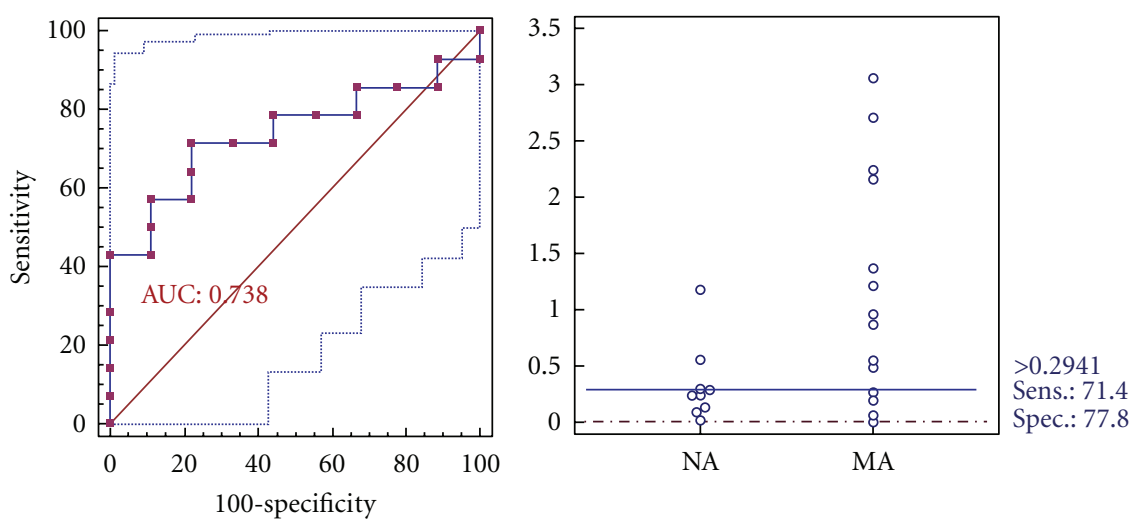

(b)

ROC curve

Serotransferrin_AVGNLR_2+/y4 (315.2/459.3)

Serotransferrin_AVGNLR_2+ /y4 (315.2/459.3)

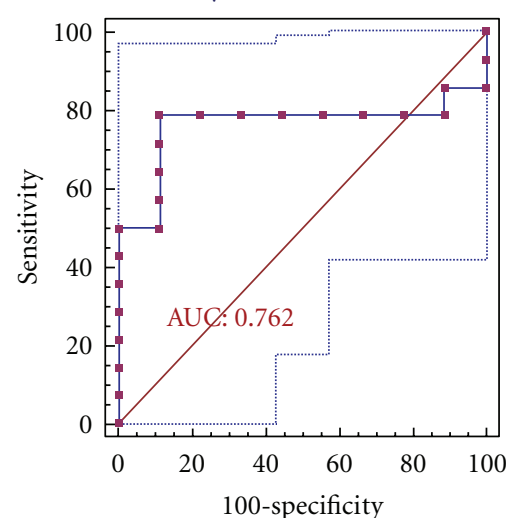

Serotransferrin AVGNLR 2+ /y4 (315.2/459.3)

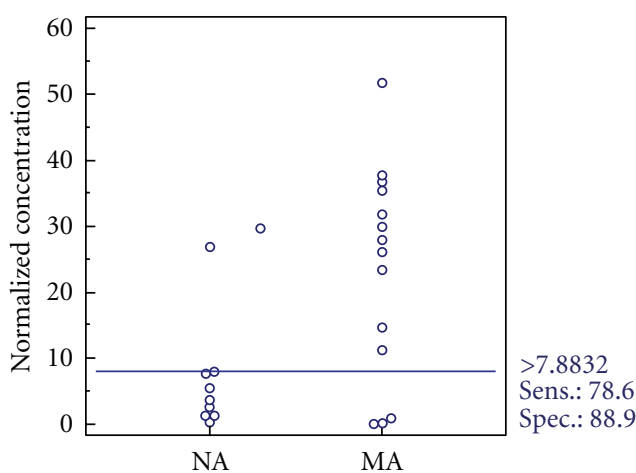

(c)

FIGURE 10 
Ceruloplasmin (transition 1-2)

\section{\{SAITRIXX)\} Mascot Search Results}

\section{Peptide View}

MS MS Fragmentation of GAYPLSIEPIGVR

Found in CERU_HUMAN, Ceruloplasmin OS=Homo sapiens $\mathrm{GN}=\mathrm{CP} P \mathrm{PE}=1 \mathrm{SV}=$

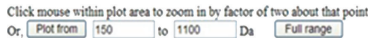

Label all possible matches 0 Label macties ssed for scoring ${ }^{\circ}$
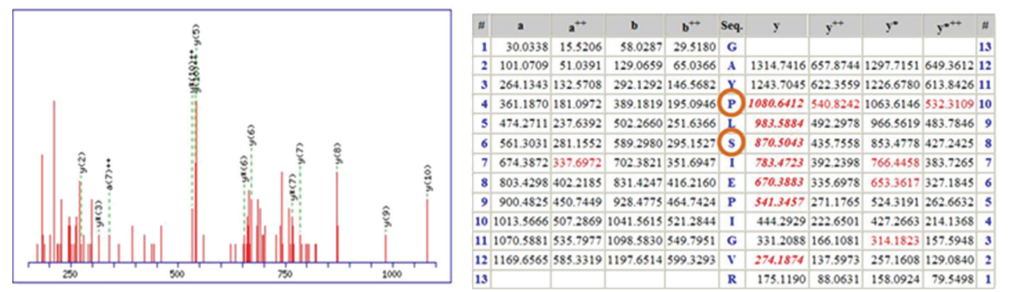

(a)

ROC curve

Ceruloplasmin_GAYPLSIEPIGVR_2+/y10 (686.4/1080.6)

Ceruloplasmin_GAYPLSIEPIGVR_2+ /y10 (686.4/1080.6)

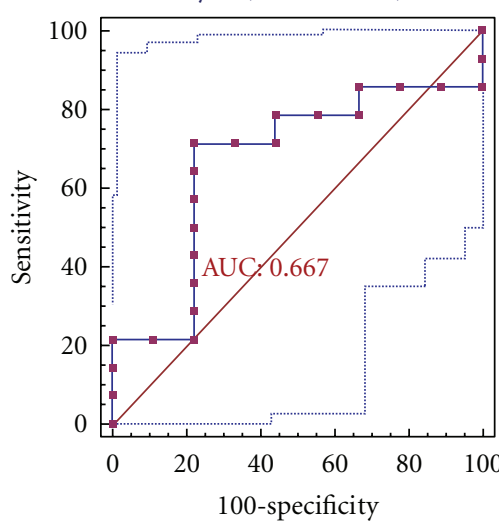

Ceruloplasmin_GAYPLSIEPIGVR_2+ /y10 (686.4/1080.6)

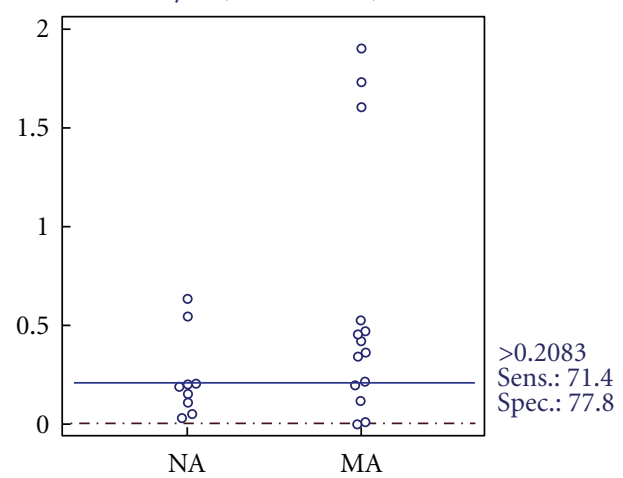

(b)

ROC curve

Ceruloplasmin_GAYPLSIEPIGVR_2+/y8 (686.4/870.5)

Ceruloplasmin_GAYPLSIEPIGVR_2+ /y8 (686.4/870.5)

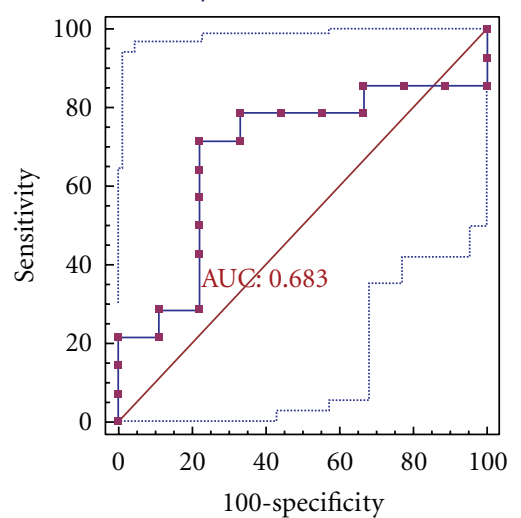

Ceruloplasmin_GAYPLSIEPIGVR_2+ /y8 (686.4/870.5)

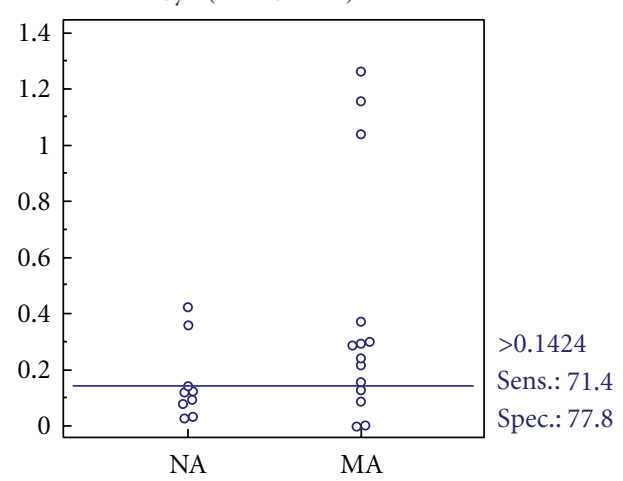

(c)

Figure 11 
Alpha-1-antitrypsin (transition 1-2)

\section{\{MAIRNCXX\} Mascot Search Results}

Peptide View

MS MS Fragmentation of LSITGTYDLK

Found in A1AT HUMAN, Alpha-1-antitopsin OS=Homo sapiens GN=SERPNA1 PE=1 SV=3

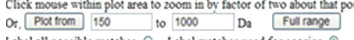

Latel all posisile matches 0 Label matches used for scoring
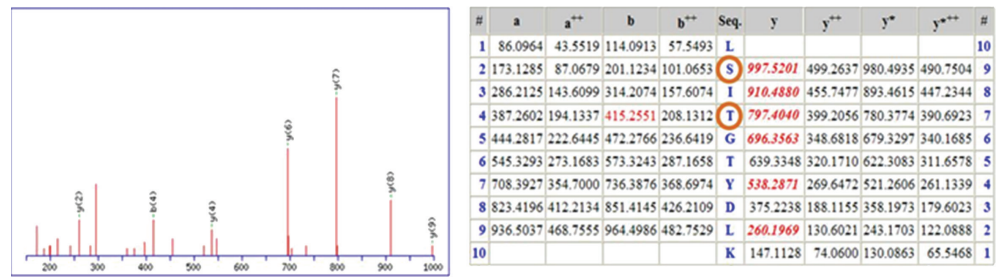

(a)

ROC curve

Alpha-1-antitrypsin_LSITGTYDLK_2+/y9 (555.8/997.5)

Alpha-1-antitrypsin

LSITGTYDLK_2+/y9 (555.8/997.5)

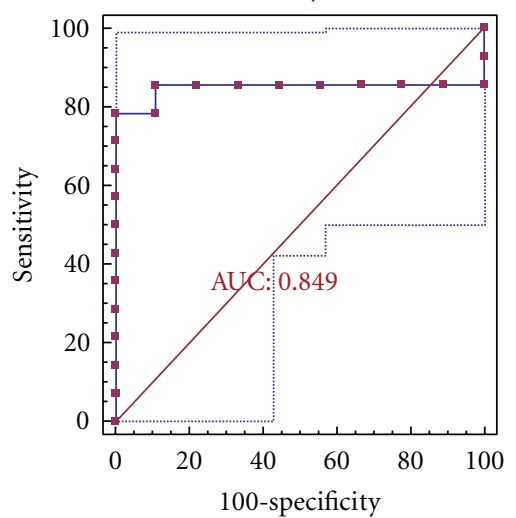

Alpha-1-antitrypsin LSITGTYDLK_2+/y9 (555.8/997.5)

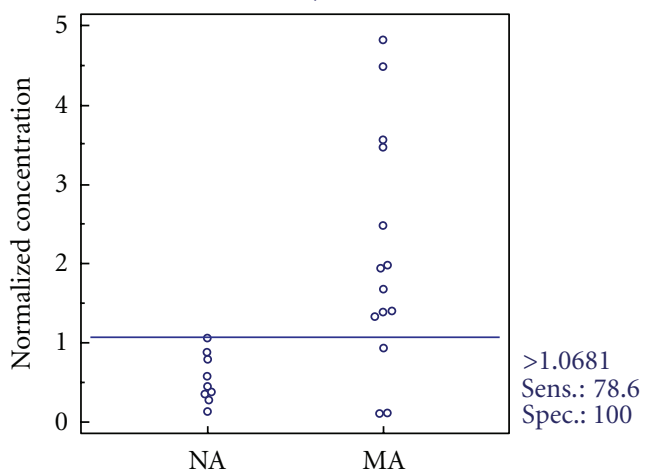

(b)

ROC curve

Alpha-1-antitrypsin_LSITGTYDLK_2+/y7 (555.8/797.4)

Alpha-1-antitrypsin_

LSITGTYDLK_2+/y7 (555.8/797.4)

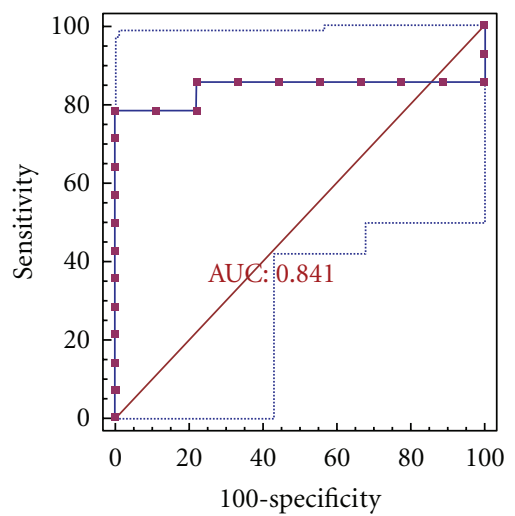

Alpha-1-antitrypsin LSITGTYDLK_2+/y7 (555.8/797.4)

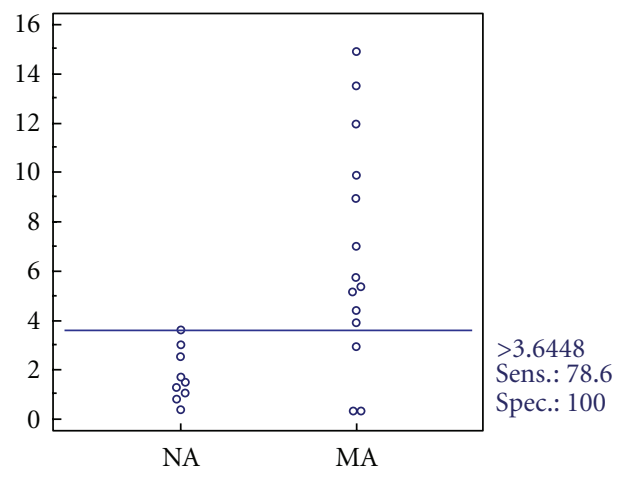

(c)

Figure 12 
Alpha-1-antitrypsin (transition 3-4)

\section{${ }_{\{S C I E N C E X\}}^{\{M A T I X)}$ Mascot Search Results}

Peptide View

MSMS Fragmentation of VVNPTQK

Found in A1AT HCMAN, Alpha-1-antitrypsin OS=Homo sapiens $\mathrm{GN}=S E R P I N A 1 \mathrm{PE}=1 \mathrm{SV}=3$

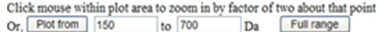

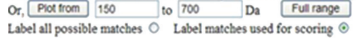

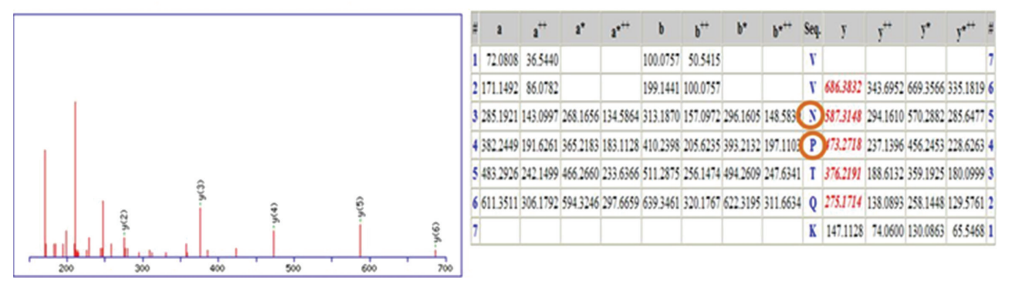

(a)

ROC curve

Alpha-1-antitrypsin_VVNPTQK_2+/y5 (393.2/587.3)

Alpha-1-antitrypsin_VVNPTQK_2+

/y5 (393.2/587.3)

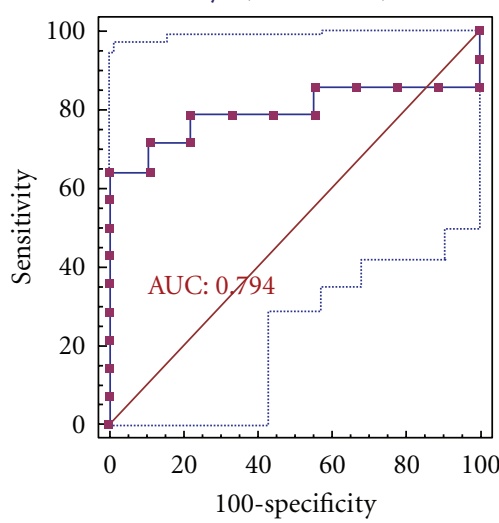

Alpha-1-antitrypsin_VVNPTQK_2+ ly5 (393.2/587.3)

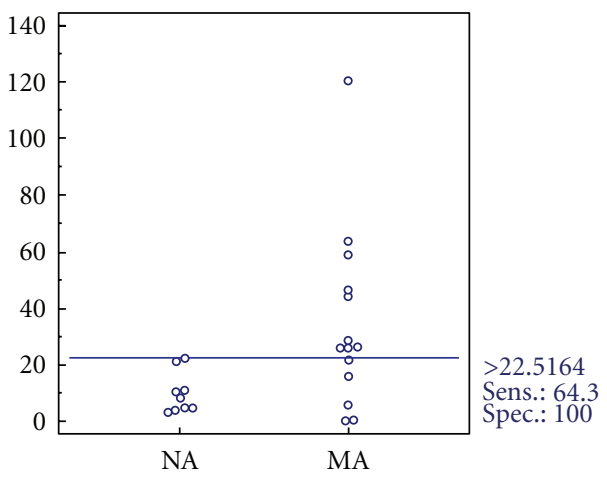

(b)

Alpha-1-antitrypsin_VVNPTQK_2+/y4 (393.2/473.3)

Alpha-1-antitrypsin_VVNPTQK_2+ /y4 (393.2/473.3)

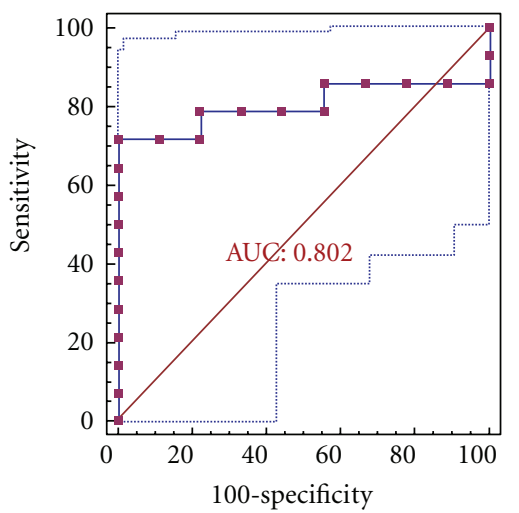

Alpha-1-antitrypsin_VVNPTQK_2+ /y4 (393.2/473.3)

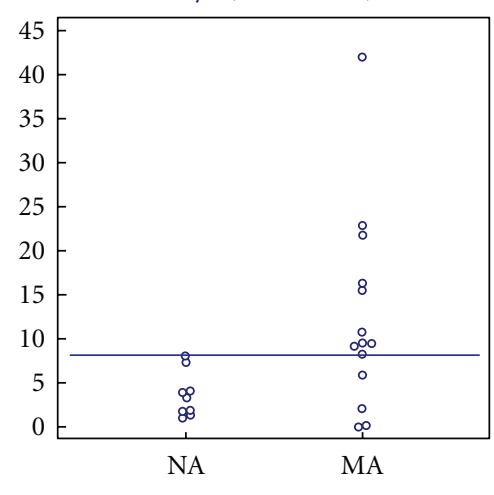

$>8.171$

Sens.: 71.4

Spec.: 100

(c)

Figure 13 
Vitamin D-binding protein (transition 1-2)

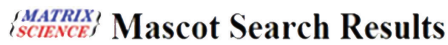

Peptide View

MS MS Fragmentation of VLEPTLK

Found in VTDB_HUMAN, Vitamin D-binding protein OS=Homo sapiens $\mathrm{GN}=\mathrm{GC} \quad \mathrm{PE}=1 \mathrm{SV}=1$

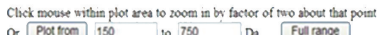

Latel all possible maxcbes 0 Luel maches used for yoring 0

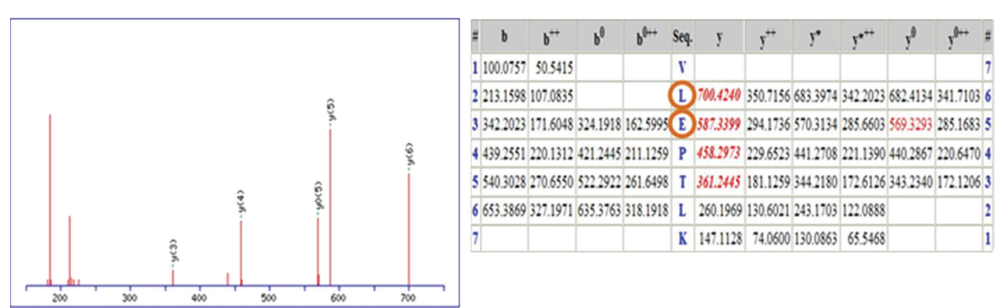

(a)

ROC curve

Vitamin D-binding protein_VLEPTLK_2+/y6 (400.2/700.4)

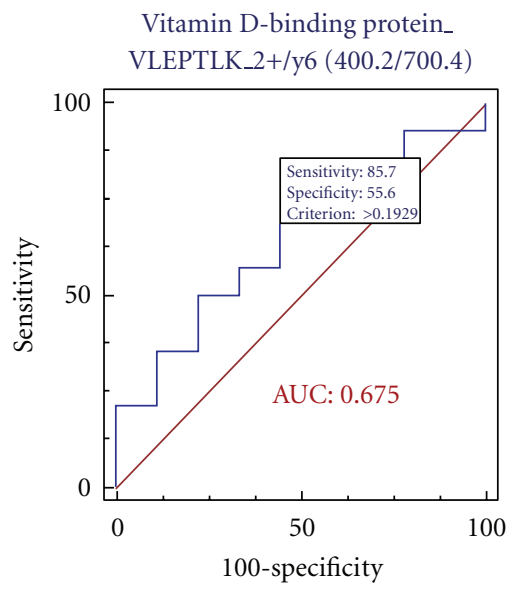

Vitamin D-binding protein

VLEPTLK_2+/y6 (400.2/700.4)

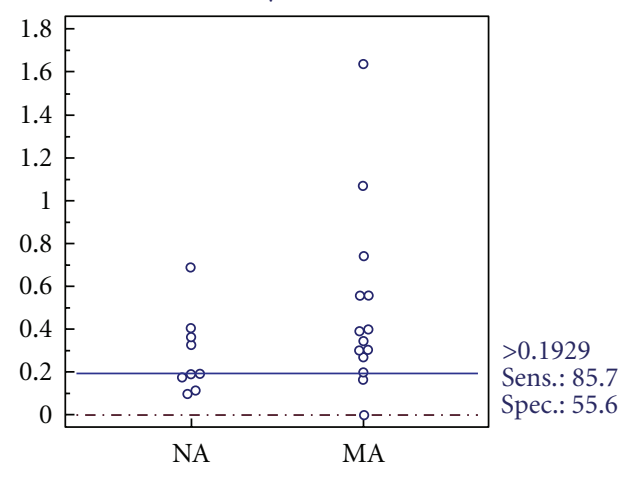

(b)

ROC curve

Vitamin D-binding protein_VLEPTLK_2+/y5 (400.2/587.3)

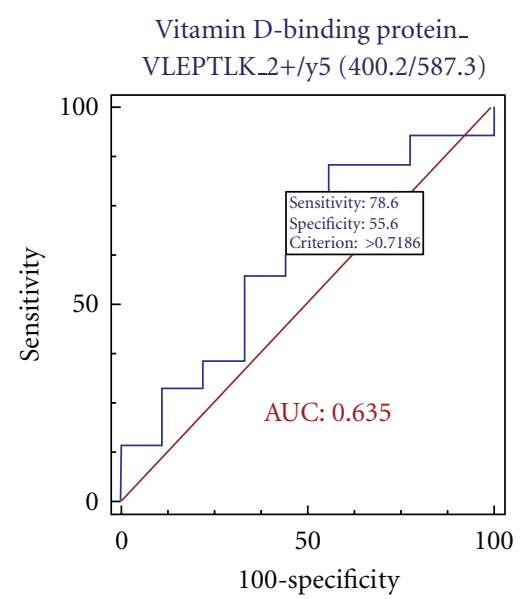

Vitamin D-binding protein VLEPTLK_2+/y5 (400.2/587.3)

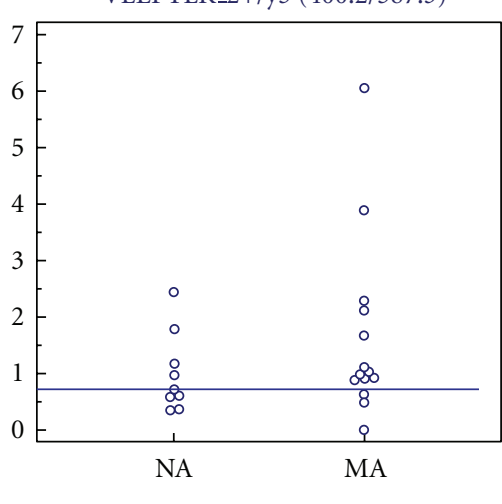

$>0.7186$

Sens.: 78.6

Spec.: 55.6

(c)

FIGURE 14 
Alpha-1-acid glycoprotein 1 (transition 1)

\section{$\left.{ }_{\{S C I R N C E X X)}^{S A T R}\right\}$ Mascot Search Results}

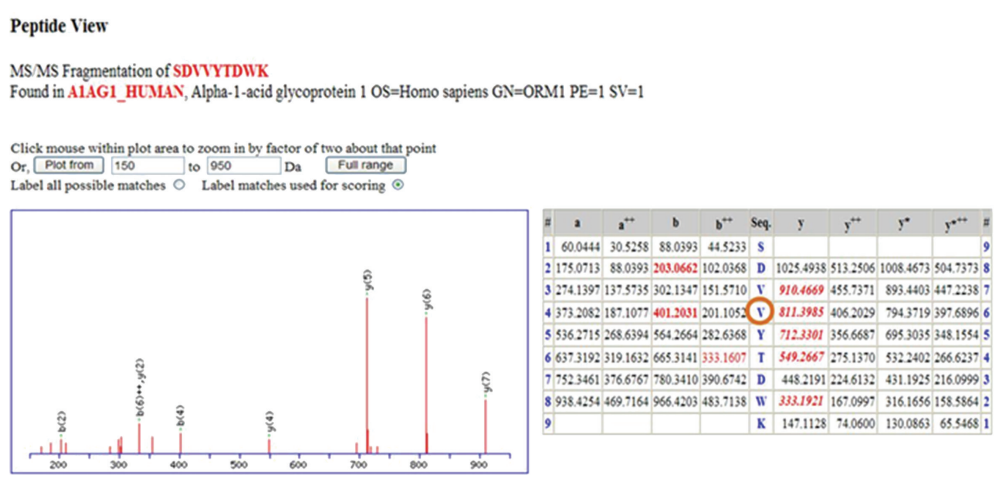

ROC curve

Alpha-1-acid glycoprotein 1_SDVVYTDWK_2+/y6 (556.8/811.4)

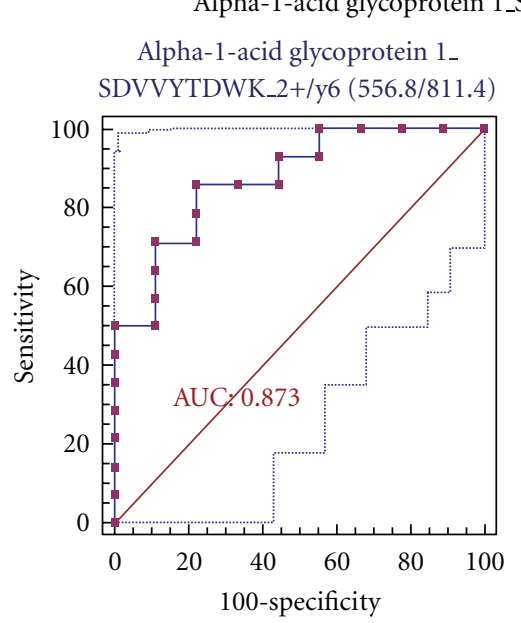

Alpha-1-acid glycoprotein 1 SDVVYTDWK_2+/y6 (556.8/811.4)

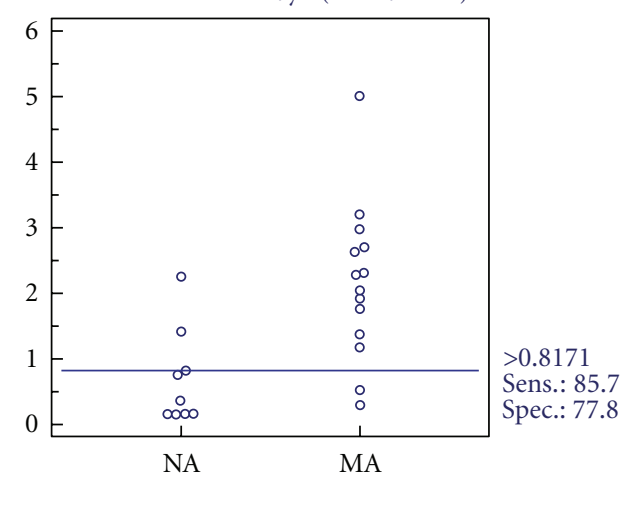

Figure 15

[38]. If reabsorption is impaired, however, these proteins can be overexcreted into urine.

$V D B P$ is a multifunctional serum glycoprotein and is an important mediator in immunopathogenesis because it is associated with immunoglobulin receptors on the surfaces of B- and T-lymphocytes [12]. Moreover, VDBP binds to circulating vitamin D metabolites with high affinity [39], and $V D B P$-bound 25-hydroxyvitamin D3 crosses the GBM and is reabsorbed by proximal tubular cells in a megalin-dependent manner, suggesting that it controls renal uptake and the activation of metabolites $[4,40]$. VDBP, also listed in the UPB database, was upregulated and is associated with diabetic nephropathy and Dents disease. In our iTRAQ and Western blot, $V D B P$ was commonly upregulated in the comparison of microalbuminuric and normoalbuminuric urinary proteome (Table 2).

$A G P 1$ is synthesized in response to systemic tissue injury, inflammation, and infection, like most other acute phase proteins [7, 41]. Further, increased levels of AGP1 reflect elevated levels of cytokines, such as interleukin-1 (IL-1), IL-6, and tumor necrosis factor alpha (TNF- $\alpha$ ), which are associated with type 2 diabetes [7]. A study has demonstrated that $A G P 1$ is an indicator of tubular disorder in multiple myeloma [42], and another has shown that serum and urinary AGP1 levels are elevated in type 2 diabetic patients with kidney disorders [5, 7]. AGP1 was listed in the UPB database, showing upregulation, and is associated with diabetic nephropathy, diabetic kidney disorder, preeclampsia, and acute appendicitis. In our iTRAQ and Western blot, AGP1 was also commonly upregulated in the comparison of microalbuminuric and normoalbuminuric urinary proteome (Table 2).

$F A B P$ (fatty acid-binding protein: $F A B P$ ) correlated with other types of diseases [43] and was also chosen for further validation. FABP was not listed in the UPB database, and in this iTRAQ and 2-DE, FABP was commonly downregulated in the comparison of microalbuminuric and normoalbuminuric urinary proteome (Table 2).

PSCA is highly expressed in the prostate and, to a lesser extent, in the bladder, placenta, colon, kidney, and stomach. Moreover, it is upregulated in prostate cancer and is detected in cancers of the bladder and pancreas [44]. PSCA was not listed in the UPB database but was upregulated in the microalbuminuric versus normoalbuminuric urinary proteome in 
Alpha-1-acid glycoprotein 1 (transition 2-3)

\section{$\left.{ }_{\{S C I E N C E X\}}^{\{M A R X}\right\}$ Mascot Search Results}

Peptide View

MSMS Fragmentation of WFYLASAFR

Found in ALAG1_HUMAN, Alpha-1-acid glycoprotein 1 OS=Homo sapiens GN=ORM1 PE=1 SV=1

Click mouse within plot area to zoom in by factor of two about that point
Or. Plot from 150 to 1050 Da Full range

Label all possible matches $O$ to Label matches used for scoring $\odot$

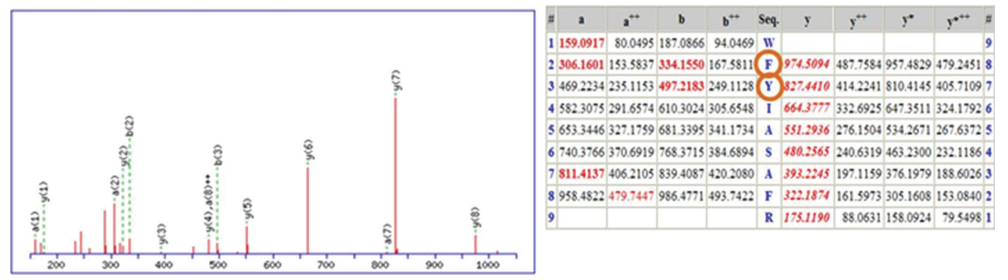

(a)

ROC curve

Alpha-1-acid glycoprotein 1_WFYIASAFR_2+/y8 (580.8/974.5)

Alpha-1-acid glycoprotein 1

WFYIASAFR_2+/y8 (580.8/974.5)

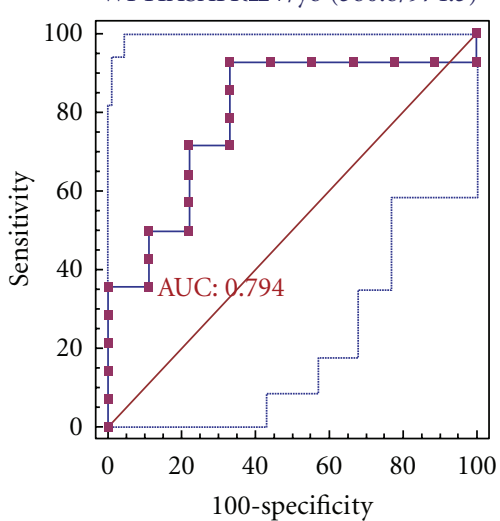

Alpha-1-acid glycoprotein 1

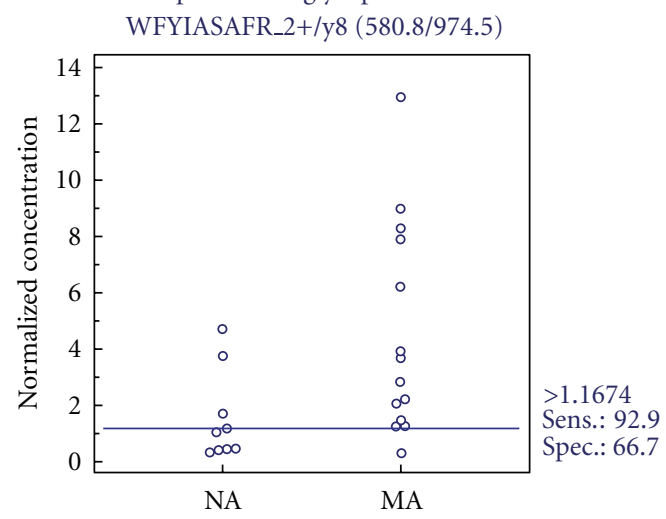

(b)

ROC curve

Alpha-1-acid glycoprotein 1_WFYIASAFR_2+/y7 (580.8/827.4)

Alpha-1-acid glycoprotein $1_{-}$

WFYIASAFR_2+/y7 (580.8/827.4)

Alpha-1-acid glycoprotein 1

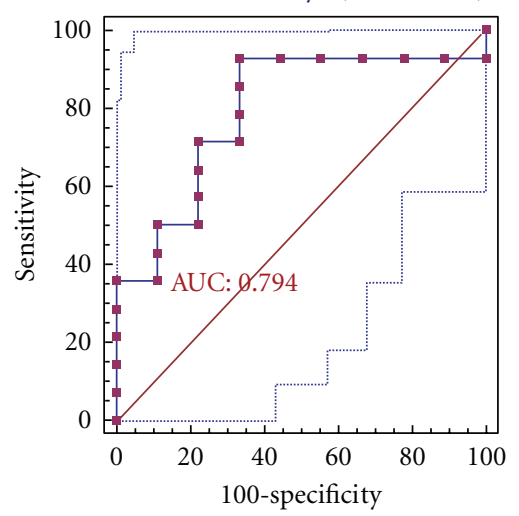
WFYIASAFR_2+/y7 (580.8/827.4)

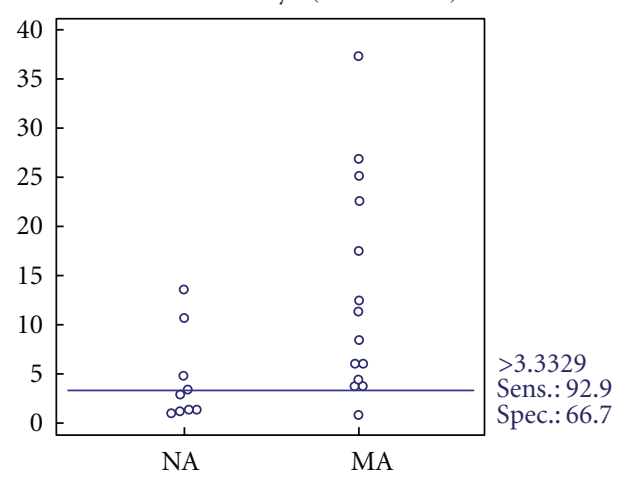

(c)

Figure 16 
Haptoglobin (transition 1)

\section{$\left\{\begin{array}{l}\text { MATRIX }\} \\ \text { SCIENCE }\end{array}\right.$ Mascot Search Results}

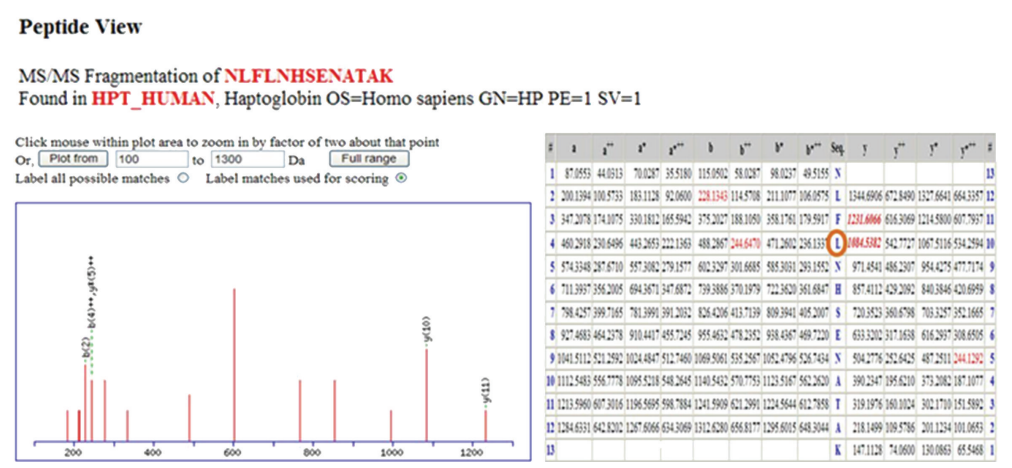

ROC curve

Haptoglobin_NLFLNHSENATAK_2+/y10 (729.9/1084.5)

Haptoglobin_NLFLNHSENATAK_ $2+/ y 10(729.9 / 1084.5)$

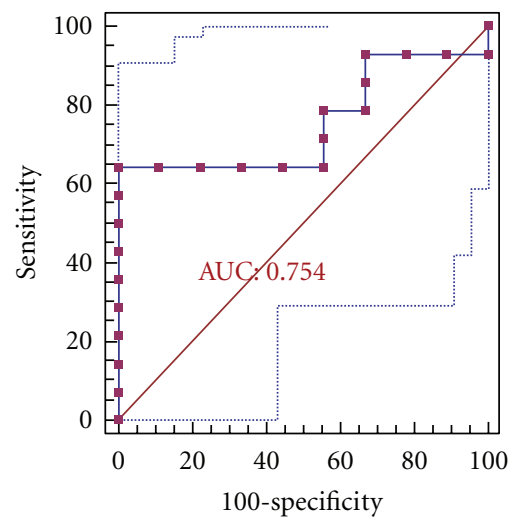

Haptoglobin_NLFLNHSENATAK_ $2+/$ y10 $(729.9 / 1084.5)$

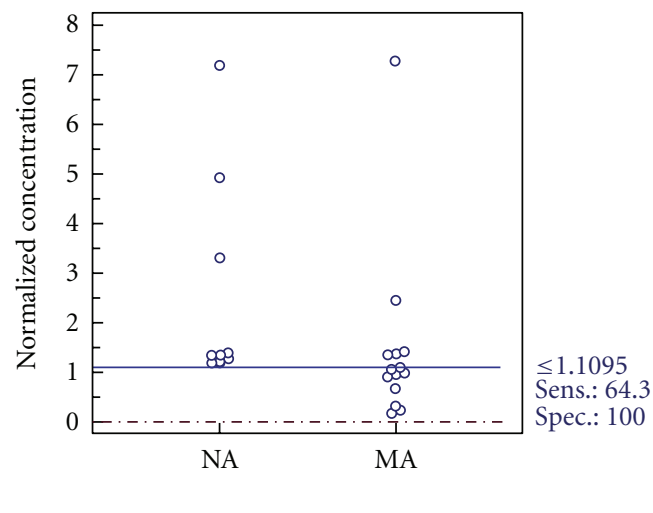

Figure 17

our ITRAQ experiment (Table 2). No relationship between prostate stem cell antigen and diabetic nephropathy has been reported.

4.4. Validation of Differentially Excreted Proteins Using MRM. For the MRM experiments, we used a bacterial beta galactosidase peptide as the internal standard for relative quatitation [28]. Seven preliminary biomarker candidates ( $T F, C P$, $A 1 A T, V D B P, A G P 1, H P$, and $P S C A)$ were confirmed in 9 normoalbuminuric and 14 microalbuminuric urine samples by MRM (Figure 7).

In the interactive plots, $T F, C P, A 1 A T, V D B P, A G P 1$, and $P S C A$ were preferentially excreted in microalbuminuria versus normoalbuminuria, whereas $H P$ was downregulated. $T F, C P, A 1 A T, V D B P$, and $A G P 1$ had the same pattern of excretion in the ITRAQ and western analysis, and $H P$ had the opposite pattern between the MRM and Western analysis. $H P$ consists of $\alpha$-chain (amino acid sequence: $19-160$ ) and $\beta$ chain (aminoacid sequence: 162-406), which are connected by disulfide bridges. In the MRM experiment, the transition (amino acid sequence: 203-215) in the $\beta$-chains was used for the relative quantitation of HP. In contrast, portions of both the $\alpha$-chain and $\beta$-chain were used in the iTRAQ quantitation (sequence coverage: $31.0 \%$ ), and an antibody that targeted a sequence in the $\alpha$-chain was used for the Western blot analysis. It is conceivable that these disparate targets resulted in contradictory patterns between iTRAQ, Western blot, and MRM. Regardless of the methods or targets, reproducible patterns must be obtained with each method.

Furthermore, we performed a multiplex assay to improve AUC values with 3 biomarker candidates (alpha-1-antitrypsin, alpha-1-acid glycoprotein 1 , and prostate stem cell antigen), obtaining a merged AUC value of 0.921 , which is greater than those of the individual proteins $(0.849,0.873$, and 0.825 for alpha-1-antitrypsin, alpha-1-acid glycoprotein 1 , and prostate stem cell antigen, resp.) (Figure 8).

Although our results require further validation in a larger collection of urine samples that contains various control samples, it appears that A1AT, AGP1, and PSCA are excellent biomarker candidates, with AUC values $>0.8$; combining the candidates improved the AUC value of 0.921. Accordingly, the other differentially expressed proteins from our iTRAQ experiment in Appendix A are potential candidates for further validation in obtaining DN biomarkers. 
Haptoglobin (transition 2)

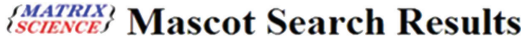

\section{Peptide View}

MS/MS Fragmentation of VSVNER

Found in HPT HUMAN, Haptoglobin OS=Homo sapiens $\mathrm{GN}=\mathrm{HP} \quad \mathrm{PE}=1 \mathrm{SV}=1$

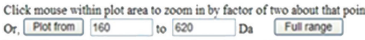

Label all possible matches $O$ Label matches used for scoring $\odot$

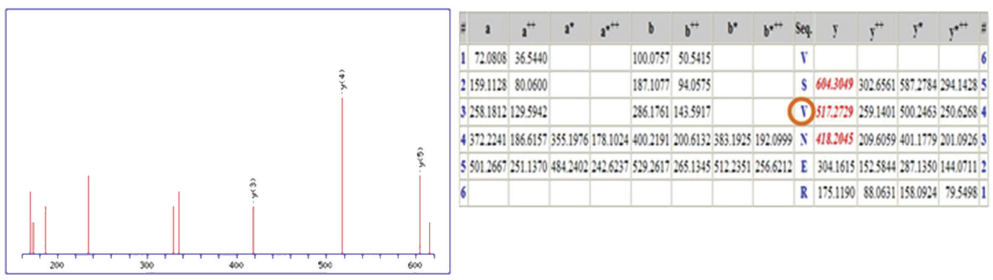

ROC curve

Haptoglobin_VSVNER_2+/y4 (352.2/517.3)
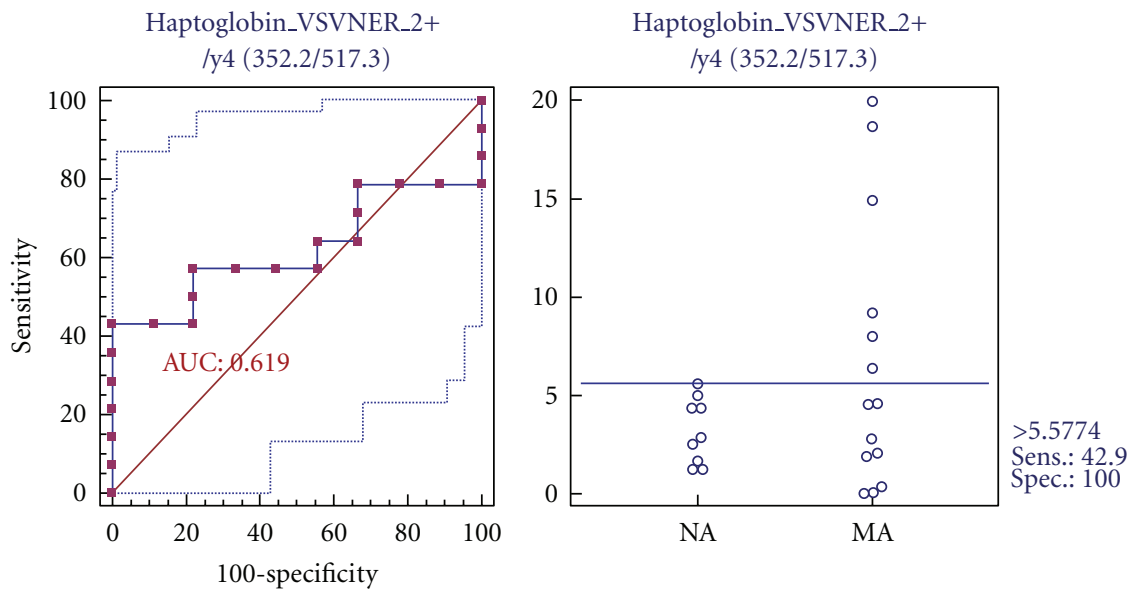

Figure 18

\section{Conclusions}

Microalbuminuria is used as a noninvasive index for the detection of diabetic renal disease. Yet, more specific and accurate biomarkers for DN are required, particularly in type 2 diabetic patients, due to several reasons, including nonspecific detection in nondiabetic renal disease, cardiovascular disease, inflammation, and hypertension. In our iTRAQ experiments, 710 urinary proteins were identified at a $>95 \%$ confidence level, of which 196 were differentially excreted by $>1.25$ or $<0.80-99$ and 97 proteins were up- and down-regulated, respectively (Appendix A).

We prioritized 196 proteins to select preliminary biomarker candidates by characterizing them with regard to "biological process" and "molecular function" and associating them with pathogenesis. Consequently, 10 proteins were selected. To confirm and validate these candidates, 2-DE, Western blot, and MRM were performed. Based on the MRM results, A1AT, AGP1, and PSCA, which had AUC values $>0.8$, are good biomarker candidates, and we improved the AUC value to 0.921 on combining the 3 proteins.
Further validation studies on other differentially excreted proteins might contribute to a greater understanding of the mechanism of renal dysfunction and its association with the pathogenesis of DN, facilitating the development of better biomarkers for $\mathrm{DN}$.

\section{Appendices}

\section{A. Differentially Excreted Urinary Proteome in Microalbumiuric versus Normoalbuminuric Urine}

For more details, see Table 5.

\section{B. Mascot Search and ROC Curves for Each Transition of MRM}

To verify the MRM validation, we analyzed minimum of 2 transitions for each biomarker candidate protein.

Results from Mascot search and ROC curves for each transition are summarized in Figures 9, 10, 11, 12, 13, 14, $15,16,17,18$, and 19 . 
Prostate stem cell antigen (transition 1-2)

\section{$\left\{\begin{array}{l}\{\text { ATRIXX } \\ \{\text { SCIENCE }\end{array}\right\}$ Mascot Search Results}

\section{Peptide View}

MS/MS Fragmentation of AVGLLTVISK

Found in gi|10720240, RecName: Full=Prostate stem cell antigen; Flags: Precursor

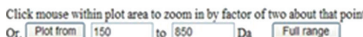

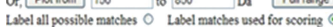
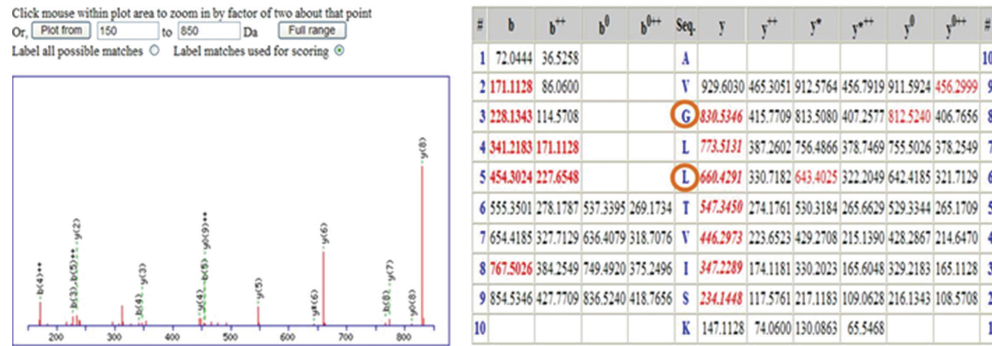

(a)

ROC curve

Prostate stem cell antigen_AVGLLTVISK_2+/y8 (501.0/830.5)

Prostate stem cell antigen AVGLLTVISK_2+/y8 (501.0/830.5)

Prostate stem cell antigen

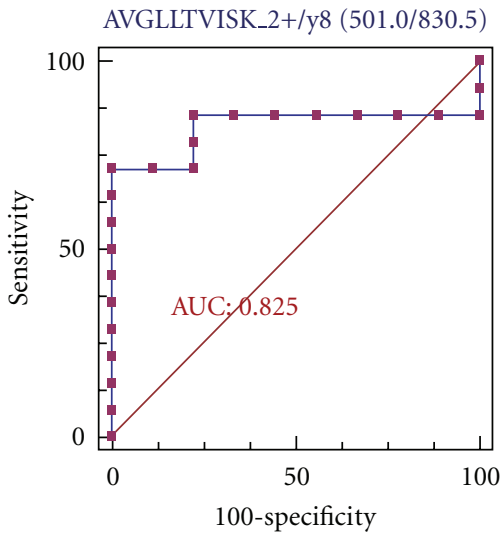
AVGLLTVISK_2+/y8 (501.0/830.5)

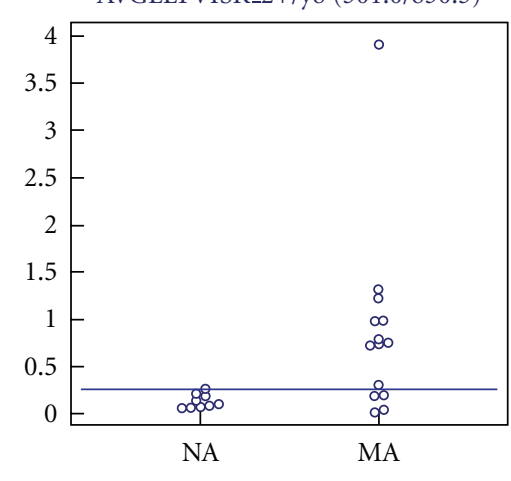

$>0.2563$

Spec.: 100

(b)

ROC curve

Prostate stem cell antigen_AVGLLTVISK_2+/y6 (501.0/660.4)

Prostate stem cell antigen AVGLLTVISK_2+/y6 (501.0/660.5)

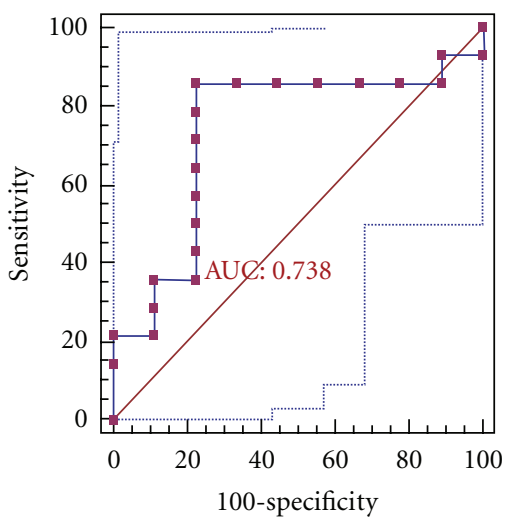

Prostate stem cell antigen AVGLLTVISK_2+/y6 (501.0/660.5)

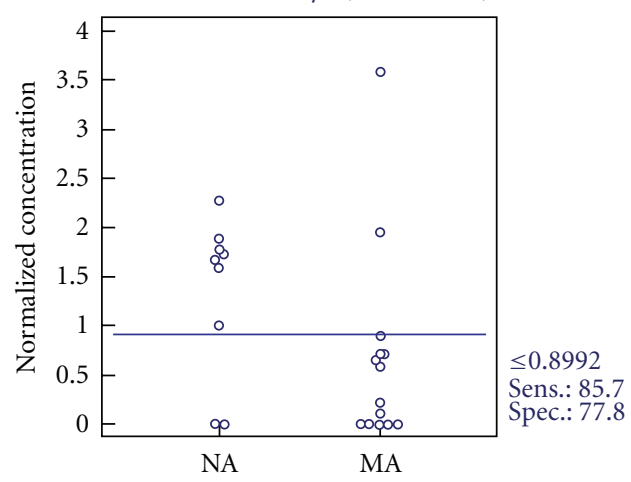

(c)

Figure 19 
TABLE 5: Differentially excreted urinary proteome in microalbumiuric versus normoalbuminuric urine.

\begin{tabular}{|c|c|c|c|c|c|c|}
\hline$N$ & Unique peptides $^{\mathrm{a}}$ & Accession number ${ }^{\mathrm{b}}$ & Protein name & Ratio $^{c}$ MA : NA & Pvald $^{\text {d }}$ A : NA & $\mathrm{EF}^{\mathrm{e}} \mathrm{MA}: \mathrm{NA}$ \\
\hline 1 & 651 & spt|P02768 & Serum albumin & 3.09 & 0.00 & 1.04 \\
\hline 2 & 337 & $\mathrm{gb} \mid \mathrm{AAF} 01333.1$ & Serum albumin & 0.36 & 0.00 & 1.08 \\
\hline 3 & 669 & $\operatorname{trm} \mid \mathrm{Q} 8 \mathrm{~N} 4 \mathrm{~N} 0$ & Alpha-2-glycoprotein 1 & 1.48 & 0.00 & 1.02 \\
\hline 4 & 639 & rf|NP_003352.1 & uromodulin & 0.72 & 0.00 & 1.04 \\
\hline 5 & 269 & emb|CAA42438.1 & Zn-alpha2-glycoprotein & 1.80 & 0.00 & 1.06 \\
\hline 6 & 235 & spt|P98160 & HSPG & 0.68 & 0.00 & 1.04 \\
\hline 7 & 209 & spt|P01009 & Alpha-1-antitrypsin & 1.42 & 0.00 & 1.04 \\
\hline 8 & 414 & spt|P02763 & Alpha-1-acid glycoprotein 1 & 2.04 & 0.00 & 1.03 \\
\hline 9 & 265 & spt|P02788 & Serotransferrin & 2.46 & 0.00 & 1.10 \\
\hline 10 & 46 & spt|P02760 & AMBP protein & 1.44 & 0.00 & 1.12 \\
\hline 11 & 300 & spt|P07911 & Uromodulin & 0.24 & 0.00 & 1.08 \\
\hline 12 & 106 & $\operatorname{prf} \mid 765044 \mathrm{~A}$ & Ig G1 H Nie & 0.61 & 0.00 & 1.15 \\
\hline 13 & 223 & dbj|BAC85395.1 & Unnamed protein product & 1.37 & 0.00 & 1.10 \\
\hline 14 & 217 & emb|CAA29229.1 & Alpha-1-acid glycoprotein 1 & 2.29 & 0.00 & 1.11 \\
\hline 15 & 107 & trm|Q5VU27 & Heparan sulfate proteoglycan 2 & 2.00 & 0.00 & 1.18 \\
\hline 16 & 306 & spt|P07998 & Ribonuclease pancreatic & 0.80 & 0.00 & 1.08 \\
\hline 17 & 62 & spt|P41222 & Prostaglandin- $\mathrm{H} 2 \mathrm{D}$-isomerase & 1.40 & 0.00 & 1.13 \\
\hline 18 & 47 & spt|P00450 & Ceruloplasmin & 2.09 & 0.00 & 1.12 \\
\hline 19 & 117 & $\operatorname{prf} \mid 763134 \mathrm{~A}$ & Ig A1 Bur & 1.60 & 0.02 & 1.39 \\
\hline 20 & 46 & cra|hCP1909255 & Serine proteinase inhibitor & 1.52 & 0.00 & 1.07 \\
\hline 21 & 90 & spt|P10451 & Osteopontin & 0.57 & 0.00 & 1.43 \\
\hline 22 & 9 & spt|P04746 & Pancreatic alpha-amylase & 0.41 & 0.00 & 1.33 \\
\hline 23 & 39 & spt|P02749 & Beta-2-glycoprotein I & 1.37 & 0.00 & 1.07 \\
\hline 24 & 50 & trm|Q9UII8 & E-cadherin & 1.36 & 0.00 & 1.12 \\
\hline 25 & 34 & rf|NP_006112.2 & Keratin 1 & 0.60 & 0.00 & 1.16 \\
\hline 26 & 88 & spt|Q14624 & ITIH4 & 0.78 & 0.00 & 1.08 \\
\hline 27 & 21 & $\operatorname{trm} \mid \mathrm{Q} 6 \mathrm{~N} 025$ & $\mathrm{FN}$ & 1.27 & 0.01 & 1.20 \\
\hline 28 & 21 & $\operatorname{trm} \mid \mathrm{Q} 8 \mathrm{~N} 175$ & Keratin 10 & 0.67 & 0.00 & 1.27 \\
\hline 29 & 34 & emb|CAA48671.1 & Alpha1-antichymotrypsin & 1.64 & 0.00 & 1.17 \\
\hline 30 & 50 & spt|P00738 & Haptoglobin & 2.36 & 0.01 & 1.24 \\
\hline 31 & 22 & gb|AAA52014.1 & Cholesterol esterase & 0.46 & 0.00 & 1.10 \\
\hline 32 & 63 & spt|P05451 & Lithostathine 1 alpha & 1.54 & 0.00 & 1.05 \\
\hline 33 & 31 & trm|Q6PAU9 & Kininogen 1 & 0.75 & 0.00 & 1.18 \\
\hline 34 & 20 & spt|P04217 & Alpha-1B-glycoprotein & 1.86 & 0.00 & 1.31 \\
\hline 35 & 34 & spt|P05155 & Plasma protease $\mathrm{C} 1$ inhibitor & 0.75 & 0.00 & 1.12 \\
\hline 36 & 14 & $\operatorname{trm} \mid \mathrm{Q} 8 \mathrm{~N} 473$ & Alpha 1 type I collagen & 0.72 & 0.00 & 1.14 \\
\hline 37 & 139 & $\operatorname{trm} \mid$ Q6IB74 & ORM2 protein & 1.48 & 0.00 & 1.23 \\
\hline 38 & 22 & spt|Q8WZ75 & Roundabout homolog 4 & 0.34 & 0.00 & 1.23 \\
\hline 39 & 29 & spt|P02791 & Hemopexin & 1.73 & 0.01 & 1.33 \\
\hline 40 & 33 & $\operatorname{trm} \mid$ Q6LBL5 & GM2 activator protein & 1.45 & 0.00 & 1.06 \\
\hline 41 & 19 & pdb|1HP7_A & A Chain A, uncleaved alpha-1-antitrypsin & 1.76 & 0.00 & 1.18 \\
\hline 42 & 23 & spt|P55290 & Cadherin-13 & 0.76 & 0.00 & 1.13 \\
\hline 43 & 21 & $\operatorname{trm} \mid \mathrm{Q} 8 \mathrm{IZY7}$ & Poly-Ig receptor & 0.63 & 0.00 & 1.36 \\
\hline 44 & 25 & spt|P05154 & Plasma serine protease inhibitor & 0.76 & 0.00 & 1.12 \\
\hline 45 & 10 & $\operatorname{trm} \mid \mathrm{Q} 96 \mathrm{CZ9}$ & Cadherin 11 , type 2 , isoform 1 preproprotein & 0.44 & 0.00 & 1.50 \\
\hline 46 & 16 & $\mathrm{gb} \mid$ AAR84237.2 & Truncated epidermal growth factor & 0.48 & 0.00 & 1.25 \\
\hline 47 & 35 & spt|P24855 & Deoxyribonuclease I & 0.50 & 0.00 & 1.12 \\
\hline 48 & 17 & $\operatorname{trm} \mid$ Q7Z645 & Collagen, type VI, alpha 1 & 0.51 & 0.00 & 1.17 \\
\hline 49 & 15 & dbj|BAA19556.1 & Immunoglobulin light chain V-J region & 1.69 & 0.01 & 1.38 \\
\hline 50 & 33 & emb|CAA23842.1 & Unnamed protein product & 1.43 & 0.00 & 1.05 \\
\hline
\end{tabular}


Table 5: Continued.

\begin{tabular}{|c|c|c|c|c|c|c|}
\hline$N$ & Unique peptides $^{\mathrm{a}}$ & Accession number ${ }^{b}$ & Protein name & Ratio $^{\mathrm{c}} \mathrm{MA}: \mathrm{NA}$ & Pvald $^{\mathrm{d}} \mathrm{MA}: \mathrm{NA}$ & $\mathrm{EF}^{\mathrm{e}} \mathrm{MA}: \mathrm{NA}$ \\
\hline 51 & 32 & spt|P08571 & CD14 & 2.36 & 0.00 & 1.62 \\
\hline 52 & 26 & $\operatorname{trm} \mid$ Q6GMX2 & Hypothetical protein & 0.59 & 0.01 & 1.24 \\
\hline 53 & 111 & emb|CAA29873.2 & Alpha-1-acid glycoprotein 2 & 2.25 & 0.00 & 1.18 \\
\hline 54 & 7 & trm|Q8WY99 & Cathepsin C & 1.58 & 0.00 & 1.17 \\
\hline 55 & 11 & spt|Q92820 & Gamma-glutamyl hydrolase & 0.54 & 0.01 & 1.33 \\
\hline 56 & 13 & spt|P15586 & $\mathrm{N}$-acetylglucosamine-6-sulfatase & 1.30 & 0.02 & 1.23 \\
\hline 57 & 10 & gb|AAQ88523.1 & AQGV3103 & 0.79 & 0.05 & 1.26 \\
\hline 58 & 8 & $\operatorname{trm} \mid \mathrm{Q} 8 \mathrm{~N} 2 \mathrm{~F} 4$ & Hypothetical protein PSEC0200 & 0.71 & 0.01 & 1.29 \\
\hline 59 & 20 & $\operatorname{trm} \mid$ Q6MZU6 & Hypothetical protein DKFZp686C15213 & 0.39 & 0.00 & 1.28 \\
\hline 60 & 27 & $\operatorname{trm} \mid$ Q6LDS3 & APS protein & 1.34 & 0.00 & 1.08 \\
\hline 61 & 9 & pdb|1L9X_D & Structure Of Gamma-Glutamyl Hydrolase & 0.69 & 0.00 & 1.12 \\
\hline 62 & 15 & spt|P07339 & Cathepsin D & 1.38 & 0.01 & 1.26 \\
\hline 63 & 10 & spt|P51884 & Lumican & 0.78 & 0.01 & 1.20 \\
\hline 64 & 130 & dbj|BAC85483.1 & Unnamed protein product & 0.73 & 0.00 & 1.14 \\
\hline 65 & 9 & pdb|1ATH_B & B Chain B, Antithrombin Iii & 1.29 & 0.00 & 1.17 \\
\hline 66 & 15 & rf|NP_001822.2 & Clusterin isoform 1 & 0.63 & 0.00 & 1.25 \\
\hline 67 & 23 & $\operatorname{trm|Q5VW91~}$ & Decay accelerating factor for complement & 1.26 & 0.00 & 1.09 \\
\hline 68 & 7 & spt|P54802 & Alpha-N-acetylglucosaminidase & 0.50 & 0.00 & 1.25 \\
\hline 69 & 12 & spt|Q16270 & IGFBP-7 & 0.79 & 0.00 & 1.14 \\
\hline 70 & 8 & $\operatorname{trm} \mid$ Q5VZE3 & Golgi phosphoprotein 2 & 0.38 & 0.02 & 1.46 \\
\hline 71 & 10 & spt|P05543 & Thyroxine-binding globulin & 1.27 & 0.00 & 1.11 \\
\hline 72 & 9 & spt|P02774 & Vitamin D-binding protein & 2.44 & 0.00 & 1.15 \\
\hline 73 & 63 & rf|NP_000573.1 & Secreted phosphoprotein 1 & 0.58 & 0.00 & 1.24 \\
\hline 74 & 56 & spt|P02671 & Fibrinogen alpha/alpha-E chain & 0.67 & 0.00 & 1.11 \\
\hline 75 & 10 & trm|Q9UBG3 & Tumor-related protein & 0.32 & 0.00 & 1.67 \\
\hline 76 & 15 & $\operatorname{trm} \mid \mathrm{O} 00391$ & Quiescin Q6 & 0.77 & 0.03 & 1.25 \\
\hline 77 & 36 & $\operatorname{trm} \mid \mathrm{Q} 5 \mathrm{VY} 30$ & Retinol binding protein 4 , plasma & 1.38 & 0.00 & 1.04 \\
\hline 78 & 8 & rf|NP_004675.2 & SPARC-like 1 & 0.55 & 0.03 & 1.70 \\
\hline 79 & 9 & spt|Q92692 & Herpesvirus entry mediator B & 0.50 & 0.00 & 1.31 \\
\hline 80 & 39 & trm|Q96FE7 & HGFL protein & 0.73 & 0.00 & 1.15 \\
\hline 81 & 6 & spt|P43652 & Afamin & 4.67 & 0.00 & 1.33 \\
\hline 82 & 24 & pdb|1QDD_A & Lithostathine & 1.73 & 0.00 & 1.20 \\
\hline 83 & 24 & spt|P10153 & Nonsecretory ribonuclease & 0.83 & 0.01 & 1.13 \\
\hline 84 & 8 & spt|P16278 & Beta-galactosidase & 1.50 & 0.00 & 1.20 \\
\hline 85 & 7 & $\operatorname{trm} \mid \mathrm{Q} 5 \mathrm{VYK} 1$ & Collagen, type XII, alpha 1 & 0.75 & 0.00 & 1.15 \\
\hline 86 & 9 & emb|CAA37914.1 & Precursor (AA-19 to 692) & 1.91 & 0.01 & 1.62 \\
\hline 87 & 15 & $\operatorname{trm} \mid \mathrm{Q} 7 \mathrm{Z} 5 \mathrm{~L} 0$ & Unnamed secretory protein & 0.56 & 0.00 & 1.31 \\
\hline 88 & 11 & spt|P08236 & Beta-glucuronidase & 1.33 & 0.00 & 1.12 \\
\hline 89 & 13 & cra|hCP51001.2 & superoxide dismutase 3 & 0.45 & 0.00 & 1.38 \\
\hline 90 & 17 & pir|S13195 & Ganglioside M2 activator protein & 1.33 & 0.00 & 1.18 \\
\hline 91 & 12 & cra|hCP1858145 & Protein C receptor, endothelial & 1.42 & 0.00 & 1.22 \\
\hline 92 & 12 & trm|Q6IAT8 & B2M protein & 1.48 & 0.00 & 1.08 \\
\hline 93 & 7 & trm|Q9Y5X6 & Glutamate carboxypeptidase & 1.47 & 0.00 & 1.04 \\
\hline 94 & 13 & spt|P06702 & Calgranulin B & 0.41 & 0.00 & 1.36 \\
\hline 95 & 11 & emb|CAB90482.1 & Human type XVIII collagen & 0.56 & 0.01 & 1.48 \\
\hline 96 & 7 & $\operatorname{trm} \mid \mathrm{O} 00533$ & Neural cell adhesion molecule & 0.73 & 0.02 & 1.29 \\
\hline 97 & 72 & spt|P04745 & Salivary alpha-amylase & 1.25 & 0.00 & 1.12 \\
\hline 98 & 40 & spt|O75594 & Peptidoglycan recognition protein & 0.56 & 0.00 & 1.08 \\
\hline 99 & 127 & emb|CAA40946.1 & Immunoglobulin lambda light chain & 1.71 & 0.01 & 1.47 \\
\hline 100 & 15 & trm|Q9UJ36 & Transmembrane glycoprotein & 0.74 & 0.01 & 1.25 \\
\hline 101 & 4 & gb|AAH17802.1 & SPRR3 protein & 0.14 & 0.00 & 1.25 \\
\hline 102 & 9 & spt|Q01469 & Fatty acid-binding protein & 0.29 & 0.00 & 1.34 \\
\hline
\end{tabular}


Table 5: Continued.

\begin{tabular}{|c|c|c|c|c|c|c|}
\hline$N$ & Unique peptides $^{\mathrm{a}}$ & Accession number ${ }^{b}$ & Protein name & Ratio $^{\mathrm{c}} \mathrm{MA}$ : NA & $\mathrm{Pval}^{\mathrm{d}} \mathrm{MA}: \mathrm{NA}$ & $\mathrm{EF}^{\mathrm{e}} \mathrm{MA}: \mathrm{NA}$ \\
\hline 103 & 4 & $\operatorname{trm} \mid$ Q6FGL5 & LCN2 protein & 1.36 & 0.01 & 1.33 \\
\hline 104 & 10 & $\operatorname{trm} \mid$ Q9UMV3 & MBL-associated serine protease 2 & 0.29 & 0.00 & 1.34 \\
\hline 105 & 10 & gb|АAH30653.1 & Cadherin 13, preproprotein & 2.91 & 0.00 & 1.76 \\
\hline 106 & 6 & spt|Q9H8L6 & Multimerin 2 & 0.69 & 0.02 & 1.34 \\
\hline 107 & 33 & $\operatorname{trm} \mid$ Q9UD19 & Intron-containing kallikrein & 0.73 & 0.02 & 1.30 \\
\hline 108 & 4 & spt|P07195 & L-lactate dehydrogenase B chain & 0.75 & 0.00 & 1.16 \\
\hline 109 & 9 & spt|P08185 & Corticosteroid-binding globulin & 3.22 & 0.00 & 1.79 \\
\hline 110 & 14 & $\operatorname{trm} \mid$ Q5UGI3 & Ubiquitin C splice variant & 1.37 & 0.01 & 1.24 \\
\hline 111 & 6 & pdb $\mid$ 1O1P_D & D Chain D, Deoxy Hemoglobin & 0.55 & 0.00 & 1.32 \\
\hline 112 & 7 & spt|P80723 & Brain acid soluble protein 1 & 2.24 & 0.00 & 1.46 \\
\hline 113 & 7 & spt|P19320 & Vascular cell adhesion protein 1 & 1.25 & 0.03 & 1.23 \\
\hline 114 & 6 & spt|P27797 & Calreticulin & 1.39 & 0.00 & 1.13 \\
\hline 115 & 10 & spt|Q01459 & Di-N-acetylchitobiase & 1.43 & 0.01 & 1.26 \\
\hline 116 & 6 & trm|Q6PN97 & Alpha 2 macroglobulin & 3.04 & 0.00 & 1.84 \\
\hline 117 & 5 & $\mathrm{gb} \mid \mathrm{AAV} 40827.1$ & superoxide dismutase 3 & 0.48 & 0.00 & 1.15 \\
\hline 118 & 3 & spt|P08473 & Neprilysin & 2.26 & 0.00 & 1.51 \\
\hline 119 & 61 & $\operatorname{trm} \mid$ Q9Y5Y7 & LYVE-1 & 1.63 & 0.00 & 1.03 \\
\hline 120 & 2 & spt|P26038 & Moesin & 1.56 & 0.00 & 1.18 \\
\hline 121 & 9 & $\operatorname{trm} \mid \mathrm{Q} 6 \mathrm{PIJ0}$ & FCGR3A protein & 0.77 & 0.00 & 1.06 \\
\hline 122 & 7 & spt|P14209 & T cell surface glycoprotein E2 & 2.02 & 0.02 & 1.61 \\
\hline 123 & 6 & spt|P02765 & Alpha-2-HS-glycoprotein & 1.69 & 0.00 & 1.20 \\
\hline 124 & 215 & $\operatorname{pir} \mid \mathrm{A} 23746$ & Ig kappa chain V-III & 1.31 & 0.00 & 1.14 \\
\hline 125 & 3 & $\operatorname{trm} \mid$ Q9NT71 & Hypothetical protein DKFZp761A051 & 0.74 & 0.03 & 1.31 \\
\hline 126 & 6 & $\operatorname{trm} \mid \mathrm{Q} 9 \mathrm{Y} 4 \mathrm{~W} 4$ & Type XV collagen & 0.52 & 0.00 & 1.25 \\
\hline 127 & 5 & cra|hCP42501.1 & Complement component 1 & 0.74 & 0.00 & 1.20 \\
\hline 128 & 11 & spt|P09564 & T-cell antigen CD7 & 0.67 & 0.01 & 1.26 \\
\hline 129 & 6 & dbj|BAA86053.1 & Carboxypeptidase E & 0.79 & 0.02 & 1.20 \\
\hline 130 & 6 & spt|P15151 & Poliovirus receptor & 1.46 & 0.01 & 1.51 \\
\hline 131 & 13 & $\operatorname{trm} \mid \mathrm{Q} 8 \mathrm{IUP} 2$ & Protocadherin 1, isoform 1 & 0.49 & 0.02 & 1.75 \\
\hline 132 & 3 & $\operatorname{trm} \mid \mathrm{Q} 8 \mathrm{NBK} 0$ & Hypothetical protein & 1.31 & 0.01 & 1.19 \\
\hline 133 & 5 & spt|P22891 & Vitamin K-dependent protein Z & 0.76 & 0.01 & 1.21 \\
\hline 134 & 6 & $\operatorname{trm} \mid$ Q9UNF4 & Hyaluronic acid receptor & 1.50 & 0.01 & 1.29 \\
\hline 135 & 11 & $\operatorname{trm} \mid \mathrm{Q} 9 \mathrm{HCU} 0$ & Tumor endothelial marker 1 & 0.46 & 0.00 & 1.36 \\
\hline 136 & 8 & spt|P35527 & Keratin, type I cytoskeletal 9 & 0.55 & 0.00 & 1.29 \\
\hline 137 & 3 & spt|P55285 & Cadherin-6 & 0.41 & 0.00 & 1.39 \\
\hline 138 & 3 & $\operatorname{trm} \mid$ Q9BYH7 & Scavenger receptor with C-type lectin type I & 1.39 & 0.01 & 1.23 \\
\hline 139 & 2 & $\operatorname{trm} \mid \mathrm{Q} 13942$ & Calmodulin & 2.64 & 0.00 & 1.39 \\
\hline 140 & 8 & spt|P04004 & Vitronectin & 1.87 & 0.00 & 1.42 \\
\hline 141 & 11 & $\operatorname{trm} \mid \mathrm{Q} 86 Z 23$ & Hypothetical protein & 2.11 & 0.01 & 1.49 \\
\hline 142 & 140 & trm|Q9NWE3 & Hypothetical protein FLJ10084 & 1.55 & 0.00 & 1.04 \\
\hline 143 & 99 & trm|Q9NWE3 & Hypothetical protein FLJ10084 & 1.53 & 0.00 & 1.06 \\
\hline 144 & 11 & spt|P05109 & Calgranulin A & 0.42 & 0.00 & 1.34 \\
\hline 145 & 58 & trm|Q9NWE3 & Hypothetical protein FLJ10084 & 1.50 & 0.00 & 1.08 \\
\hline 146 & 3 & $\operatorname{trm|Q9BYH7~}$ & Scavenger receptor with C-type lectin type I & 1.65 & 0.00 & 1.24 \\
\hline 147 & 4 & gb|AAA52018.1 & Chromogranin A & 0.52 & 0.00 & 1.26 \\
\hline 148 & 4 & emb|CAI20248.1 & PPGB & 1.51 & 0.00 & 1.24 \\
\hline 149 & 92 & $\operatorname{pir} \mid S 12443$ & Ig lambda chain $(\mathrm{Ke}+\mathrm{O}-)$-human & 1.35 & 0.03 & 1.24 \\
\hline 150 & 7 & trm|Q5TEQ5 & OTTHUMP00000044363 & 0.29 & 0.00 & 1.58 \\
\hline
\end{tabular}


TABle 5: Continued.

\begin{tabular}{|c|c|c|c|c|c|c|}
\hline$N$ & Unique peptides $^{\mathrm{a}}$ & Accession number ${ }^{\mathrm{b}}$ & Protein name & Ratio $^{c}$ MA: NA & $\mathrm{Pval}^{\mathrm{d}} \mathrm{MA}: \mathrm{NA}$ & $\mathrm{EF}^{\mathrm{e}} \mathrm{MA}: \mathrm{NA}$ \\
\hline 151 & 18 & $\operatorname{trm} \mid$ Q6UX86 & GPPS559 & 2.08 & 0.01 & 1.60 \\
\hline 152 & 2 & $\operatorname{trm} \mid \mathrm{Q} 8 \mathrm{TCZ2}$ & MIC2L1 & 0.80 & 0.01 & 1.16 \\
\hline 153 & 5 & spt|P61970 & Nuclear transport factor 2 & 0.78 & 0.01 & 1.18 \\
\hline 154 & 284 & pdb|1T04_C & Anti-ifn-gamma fab in C2 space group & 1.45 & 0.02 & 1.28 \\
\hline 155 & 8 & spt|P00790 & Pepsin A & 0.80 & 0.00 & 1.15 \\
\hline 156 & 2 & cra|hCP1778903.1 & CD7 antigen & 0.48 & 0.00 & 1.03 \\
\hline 157 & 5 & trm|O00480 & Butyrophilin, subfamily 2 & 0.68 & 0.00 & 1.12 \\
\hline 158 & 5 & $\operatorname{trm} \mid \mathrm{O} 43653$ & Prostate stem cell A & 1.70 & 0.00 & 1.25 \\
\hline 159 & 4 & $\operatorname{trm} \mid$ Q9BX83 & Hemoglobin alpha 1 globin chain & 0.67 & 0.05 & 1.47 \\
\hline 160 & 9 & spt|P11684 & Uteroglobin & 3.16 & 0.00 & 1.86 \\
\hline 161 & 7 & $\operatorname{trm} \mid$ Q7LDY7 & Alpha-KG-E2 & 1.47 & 0.00 & 1.17 \\
\hline 162 & 3 & trm|Q9BYH7 & Scavenger receptor & 1.92 & 0.00 & 1.25 \\
\hline 163 & 4 & $\operatorname{trm} \mid \mathrm{Q} 6 \mathrm{AZK} 5$ & KRT13 protein & 0.56 & 0.02 & 1.58 \\
\hline 164 & 5 & spt|P09619 & PDGF-R-beta & 0.66 & 0.01 & 1.29 \\
\hline 165 & 5 & pdb|5TTR_H & Leu 55 Pro Transthyretin & 1.46 & 0.02 & 1.33 \\
\hline 166 & 3 & rf|NP_877418.1 & Mucin 1 , transmembrane & 0.49 & 0.02 & 1.78 \\
\hline 167 & 2 & trm|Q5SWW9 & OTTHUMP00000060590 & 0.39 & 0.01 & 1.76 \\
\hline 168 & 2 & rf|NP_003217.2 & Trefoil factor 3 & 1.63 & 0.00 & 1.22 \\
\hline 169 & 3 & spt|Q99574 & Neuroserpin & 0.63 & 0.01 & 1.36 \\
\hline 170 & 21 & trm|Q9Y3U9 & Hypothetical protein DKFZp566C243 & 0.75 & 0.00 & 1.06 \\
\hline 171 & 23 & $\mathrm{gb} \mid \mathrm{AAB} 27607.1$ & Prostaglandin D synthase & 1.20 & 0.00 & 1.10 \\
\hline 172 & 4 & $\mathrm{gb} \mid \mathrm{AAO} 11857.1$ & Immunoglobulin & 1.38 & 0.00 & 1.14 \\
\hline 173 & 2 & $\operatorname{trm} \mid \mathrm{Q} 5 \mathrm{VTA6}$ & Cubilin & 0.79 & 0.03 & 1.23 \\
\hline 174 & 3 & $\operatorname{trm} \mid \mathrm{Q} 5 S Y 67$ & OTTHUMP00000059857 & 0.69 & 0.02 & 1.33 \\
\hline 175 & 54 & spt|P15814 & Immunoglobulin lambda-like polypeptide 1 & 2.50 & 0.01 & 1.37 \\
\hline 176 & 2 & spt|P22352 & Plasma glutathione peroxidase & 0.70 & 0.00 & 1.13 \\
\hline 177 & 7 & gb|AAL68978.1 & Mutant beta globin & 0.32 & 0.00 & 1.77 \\
\hline 178 & 6 & spt|P35908 & KCytokeratin $2 \mathrm{e}$ & 0.50 & 0.03 & 1.74 \\
\hline 179 & 284 & $\mathrm{dbj} \mid \mathrm{BAB} 18261.1$ & Anti-HBs antibody light chain & 3.19 & 0.00 & 1.53 \\
\hline 180 & 3 & rf|XP_370615.2 & Hypothetical protein & 0.53 & 0.03 & 1.72 \\
\hline 181 & 164 & $\mathrm{gb} \mid \mathrm{AAB} 50880.2$ & Anitubulin IgG1 kappa VL chain & 1.64 & 0.00 & 1.30 \\
\hline 182 & 170 & dbj|BAC01692.1 & Immunoglobulin kappa light chain & 2.49 & 0.01 & 1.79 \\
\hline 183 & 2 & trm|Q7RTN9 & Type II keratin K6h & 0.43 & 0.01 & 1.52 \\
\hline 184 & 3 & spt|P21926 & Motility-related protein & 1.70 & 0.03 & 1.45 \\
\hline 185 & 2 & spt|Q13873 & Bone morphogenetic protein receptor & 0.61 & 0.01 & 1.08 \\
\hline 186 & 4 & $\mathrm{gb} \mid$ AAA62175.1 & Heat shock protein 27 & 0.53 & 0.00 & 1.38 \\
\hline 187 & 15 & spt|P02787 & transferrin & 1.86 & 0.00 & 1.25 \\
\hline 188 & 2 & spt|P07108 & Acyl-CoA-binding protein & 2.20 & 0.02 & 1.47 \\
\hline 189 & 2 & spt|Q9NZH0 & G protein-coupled receptor family $\mathrm{C}$ & 1.27 & 0.04 & 1.25 \\
\hline 190 & 2 & $\operatorname{trm} \mid$ Q96E46 & Fructose-1,6-bisphosphatase 1 & 0.44 & 0.00 & 1.01 \\
\hline 191 & 44 & gb|AAB53267.1 & Immunoglobulin V-region light chain & 1.40 & 0.03 & 1.30 \\
\hline 192 & 8 & gb|AAR32503.1 & Immunoglobulin heavy chain & 0.46 & 0.00 & 1.19 \\
\hline 193 & 7 & rf|NP_653247.1 & Immunoglobulin J chain & 0.37 & 0.01 & 1.88 \\
\hline 194 & 29 & emb|CAA12585.1 & Ig heavy chain variable region & 1.50 & 0.00 & 1.13 \\
\hline 195 & 4 & gb|AAD16731.1 & Immunoglobulin lambda light chain & 1.58 & 0.00 & 1.11 \\
\hline 196 & 33 & $\operatorname{trm} \mid$ Q6UXB8 & HGSC289 (OTTHUMP00000039678) & 1.27 & 0.04 & 1.22 \\
\hline
\end{tabular}

${ }^{a}$ The numbers of unique peptides and MS/MS spectrum observed by ProteinPilot software were determined only for those peptides with $\geq 95 \%$ confidence. ${ }^{b}$ Accession numbers represent entries in the Human CDS database (human KBMS 5.0, 2005-03-02; a total of 187,748 entries provided by Applied Biosystems).

${ }^{\mathrm{c}-\mathrm{e}}$ The iTRAQ ratio, $P$ value, and $\mathrm{EF}$ value in microalbumiuric versus normoalbuminuric urine, respectively. 


\section{Authors' Contribution}

J. Jin and Y. H. Ku contributed equally to this study.

\section{Acknowledgments}

This paper was supported by the 21C Frontier Functional Proteomics Project of the Korean Ministry of Science and Technology (Grant no. FPR 08-A2-110) and a Grant (no. 10035353) from the Seoul R\&BD Program.

\section{References}

[1] R. Klein, B. E. K. Klein, S. E. Moss, and K. J. Cruickshanks, "The wisconsin epidemiologic study of diabetic retinopathy: XVII. The 14- year incidence and progression of diabetic retinopathy and associated risk factors in type 1 diabetes," Ophthalmology, vol. 105, no. 10, pp. 1801-1815, 1998.

[2] F. P. Schena and L. Gesualdo, "Pathogenetic mechanisms of diabetic nephropathy," Journal of the American Society of Nephrology, vol. 16, supplement 1, pp. S30-S33, 2005.

[3] B. F. Schrijvers, A. S. De Vriese, and A. Flyvbjerg, "From hyperglycemia to diabetic kidney disease: the role of metabolic, hemodynamic, intracellular factors and growth factors/cytokines," Endocrine Reviews, vol. 25, no. 6, pp. 971-1010, 2004.

[4] Y. Wang, J. Zhou, A. W. Minto et al., "Altered vitamin D metabolism in type II diabetic mouse glomeruli may provide protection from diabetic nephropathy," Kidney International, vol. 70, no. 5, pp. 882-891, 2006.

[5] S. Jain, A. Rajput, Y. Kumar, N. Uppuluri, A. S. Arvind, and U. Tatu, "Proteomic analysis of urinary protein markers for accurate prediction of diabetic kidney disorder," Journal of Association of Physicians of India, vol. 53, pp. 513-520, 2005.

[6] M. Berger, D. Mönks, C. Wanner, and T. H. Lindner, "Diabetic nephropathy: an inherited disease or just a diabetic complication?" Kidney and Blood Pressure Research, vol. 26, no. 3, pp. 143-154, 2003.

[7] M. B. Gomes and V. G. Nogueira, "Acute-phase proteins and microalbuminuria among patients with type 2 diabetes," Diabetes Research and Clinical Practice, vol. 66, no. 1, pp. 3139, 2004.

[8] H. L. Hillege, V. Fidler, G. F. H. Diercks et al., "Urinary albumin excretion predicts cardiovascular and noncardiovascular mortality in general population," Circulation, vol. 106, no. 14, pp. 1777-1782, 2002.

[9] H. C. Gerstein, J. F. E. Mann, Q. Yi et al., "Albuminuria and risk of cardiovascular events, death, and heart failure in diabetic and nondiabetic individuals," Journal of the American Medical Association, vol. 286, no. 4, pp. 421-426, 2001.

[10] Y. H. Yang, S. Zhang, J. F. Cui et al., "Diagnostic potential of serum protein pattern in type 2 diabetic nephropathy," Diabetic Medicine, vol. 24, no. 12, pp. 1386-1392, 2007.

[11] H. H. Otu, H. Can, D. Spentzos et al., "Prediction of diabetic nephropathy using urine proteomic profiling 10 years prior to development of nephropathy," Diabetes Care, vol. 30, no. 3, pp. 638-643, 2007.

[12] P. V. Rao, X. Lu, M. Standley et al., "Proteomic identification of urinary biomarkers of diabetic nephropathy," Diabetes Care, vol. 30, no. 3, pp. 629-637, 2007.

[13] K. Sharma, S. Lee, S. Han et al., "Two-dimensional fluorescence difference gel electrophoresis analysis of the urine proteome in human diabetic nephropathy," Proteomics, vol. 5, no. 10, pp. 2648-2655, 2005.
[14] L. R. Zieske, "A perspective on the use of iTRAQ reagent technology for protein complex and profiling studies," Journal of Experimental Botany, vol. 57, no. 7, pp. 1501-1508, 2006.

[15] K. Aggarwal, L. H. Choe, and K. H. Lee, "Shotgun proteomics using the iTRAQ isobaric tags," Briefings in Functional Genomics and Proteomics, vol. 5, no. 2, pp. 112-120, 2006.

[16] K. Aggarwal, L. H. Choe, and K. H. Lee, "Quantitative analysis of protein expression using amine-specific isobaric tags in Escherichia coli cells expressing rhsA elements," Proteomics, vol. 5, no. 9, pp. 2297-2308, 2005.

[17] A. Glen, C. S. Gan, F. C. Hamdy et al., "ITRAQ-facilitated proteomic analysis of human prostate cancer cells identifies proteins associated with progression," Journal of Proteome Research, vol. 7, no. 3, pp. 897-907, 2008.

[18] Y. Ogata, M. C. Charlesworth, L. Higgins, B. M. Keegan, S. Vernino, and D. C. Muddiman, "Differential protein expression in male and female human lumbar cerebrospinal fluid using iTRAQ reagents after abundant protein depletion," Proteomics, vol. 7, no. 20, pp. 3726-3734, 2007.

[19] P. L. Ross, Y. N. Huang, J. N. Marchese et al., "Multiplexed protein quantitation in Saccharomyces cerevisiae using aminereactive isobaric tagging reagents," Molecular and Cellular Proteomics, vol. 3, no. 12, pp. 1154-1169, 2004.

[20] A. Matheson, M. D. P. Willcox, J. Flanagan, and B. J. Walsh, "Urinary biomarkers involved in type 2 diabetes: a review," Diabetes/Metabolism Research and Reviews, vol. 26, no. 3, pp. 150-171, 2010.

[21] M. Afkarian, M. Bhasin, S. T. Dillon et al., "Optimizing a proteomics platform for urine biomarker discovery," Molecular and Cellular Proteomics, vol. 9, no. 10, pp. 2195-2204, 2010.

[22] J. Luo, T. Ning, Y. Sun et al., "Proteomic analysis of rice endosperm cells in response to expression of HGM-CSF," Journal of Proteome Research, vol. 8, no. 2, pp. 829-837, 2009.

[23] J. Jin, J. Park, K. Kim et al., "Detection of differential proteomes of human $\beta$-cells during islet-like differentiation using iTRAQ labeling," Journal of Proteome Research, vol. 8, no. 3, pp. 1393-1403, 2009.

[24] I. V. Shilov, S. L. Seymourt, A. A. Patel et al., "The paragon algorithm, a next generation search engine that uses sequence temperature values sequence temperature values and feature probabilities to identify peptides from tandem mass spectra," Molecular and Cellular Proteomics, vol. 6, no. 9, pp. 1638-1655, 2007.

[25] A. Pierce, R. D. Unwin, C. A. Evans et al., "Eight-channel iTRAQ enables comparison of the activity of six leukemogenic tyrosine kinases," Molecular and Cellular Proteomics, vol. 7, no. 5, pp. 853-863, 2008.

[26] J. Park, S. Kim, J. K. Oh et al., "Identification of differentially expressed proteins in imatinib mesylate-resistant chronic myelogenous cells," Journal of Biochemistry and Molecular Biology, vol. 38, no. 6, pp. 725-738, 2005.

[27] J. Park, H. Kwon, Y. Kang, and Y. Kim, "Proteomic analysis of O-GlcNAc modifications derived from streptozotocin and glucosamine induced $\beta$-cell apoptosis," Journal of Biochemistry and Molecular Biology, vol. 40, no. 6, pp. 1058-1068, 2007.

[28] K. Kim, S. J. Kim, H. G. Yu et al., "Verification of biomarkers for diabetic retinopathy by multiple reaction monitoring," Journal of Proteome Research, vol. 9, no. 2, pp. 689-699, 2010.

[29] C. Shao, M. Li, X. Li et al., "A tool for biomarker discovery in the urinary proteome: a manually curated human and animal urine protein biomarker database," Molecular and Cellular Proteomics, vol. 10, no. 11, 2011. 
[30] T. Narita, M. Hosoba, M. Kakei, and S. Ito, "Increased urinary excretions of immunoglobulin G, ceruloplasmin, and transferrin predict development of microalbuminuria in patients with type 2 diabetes," Diabetes Care, vol. 29, no. 1, pp. 142-144, 2006.

[31] S. Anderson and B. M. Brenner, "Pathogenesis of diabetic glomerulopathy: hemodynamic considerations," Diabetes/ Metabolism Reviews, vol. 4, no. 2, pp. 163-177, 1988.

[32] R. Zatz, T. W. Meyer, H. G. Rennke, and B. M. Brenner, "Predominance of hemodynamic rather than metabolic factors in the pathogenesis of diabetic glomerulopathy," Proceedings of the National Academy of Sciences of the United States of America, vol. 82, no. 17, pp. 5963-5967, 1985.

[33] L. Musante, G. Candiano, M. Bruschi et al., "Characterization of plasma factors that alter the permeability to albumin within isolated glomeruli," Proteomics, vol. 2, no. 2, pp. 197-205, 2002.

[34] V. Thongboonkerd and P. Malasit, "Renal and urinary proteomics: current applications and challenges," Proteomics, vol. 5, no. 4, pp. 1033-1042, 2005.

[35] V. Thongboonkerd, M. T. Barati, K. R. McLeish et al., "Alterations in the renal elastin-elastase system in type 1 diabetic nephropathy identified by proteomic analysis," Journal of the American Society of Nephrology, vol. 15, no. 3, pp. 650-662, 2004.

[36] H. H. Y. Ngai, W. H. Sit, P. P. Jiang, V. Thongboonkerd, and J. M. F. Wan, "Markedly increased urinary preprohaptoglobin and haptoglobin in passive heymann nephritis: a differential proteomics approach," Journal of Proteome Research, vol. 6, no. 8, pp. 3313-3320, 2007.

[37] J. T. Tamsma, J. Van Den Born, J. A. Bruijn et al., "Expression of glomerular extracellular matrix components in human diabetic nephropathy: decrease of heparan sulphate in the glomerular basement membrane," Diabetologia, vol. 37, no. 3, pp. 313-320, 1994.

[38] C. Y. Hong and K. S. Chia, "Markers of diabetic nephropathy," Journal of Diabetes and its Complications, vol. 12, no. 1, pp. 4360, 1998.

[39] W. Z. Ye, D. Dubois-Laforgue, C. Bellanné-Chantelot, J. Timsit, and G. Velho, "Variations in the vitamin D-binding protein (Gc locus) and risk of type 2 diabetes mellitus in French Caucasians," Metabolism: Clinical and Experimental, vol. 50, no. 3, pp. 366-369, 2001.

[40] A. Nykjaer, D. Dragun, D. Walther et al., "An endocytic pathway essential for renal uptake and activation of the steroid 25-(OH) vitamin $\mathrm{D}_{3}$," Cell, vol. 96, no. 4, pp. 507-515, 1999.

[41] T. Fournier, N. Medjoubi-N, and D. Porquet, "Alpha-1-acid glycoprotein," Biochimica et Biophysica Acta, vol. 1482, no. 1-2, pp. 157-171, 2000.

[42] A. Corso, G. Serricchio, P. Zappasodi et al., "Assessment of renal function in patients with multiple myeloma: the role of urinary proteins," Annals of Hematology, vol. 78, no. 8, pp. 371-375, 1999.

[43] E. A. Reece, I. Ji, Y. K. Wu, and Z. Zhao, "Characterization of differential gene expression profiles in diabetic embryopathy using DNA microarray analysis," American Journal of Obstetrics and Gynecology, vol. 195, no. 4, pp. 1075-1080, 2006.

[44] Z. Gu, G. Thomas, J. Yamashiro et al., "Prostate stem cell antigen (PSCA) expression increases with high gleason score, advanced stage and bone metastasis in prostate cancer," Oncogene, vol. 19, no. 10, pp. 1288-1296, 2000. 


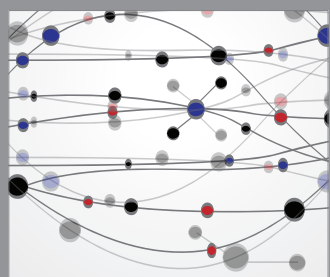

The Scientific World Journal
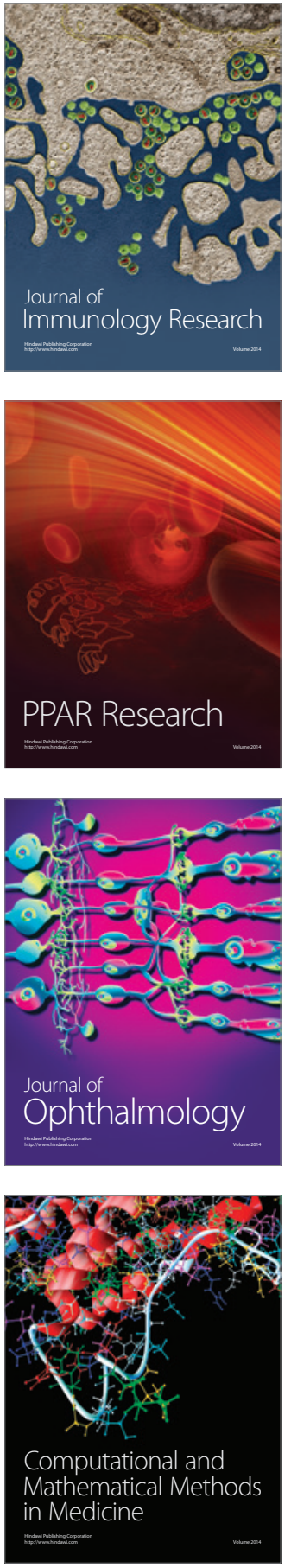

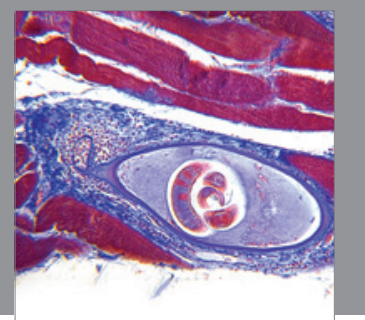

Gastroenterology

Research and Practice
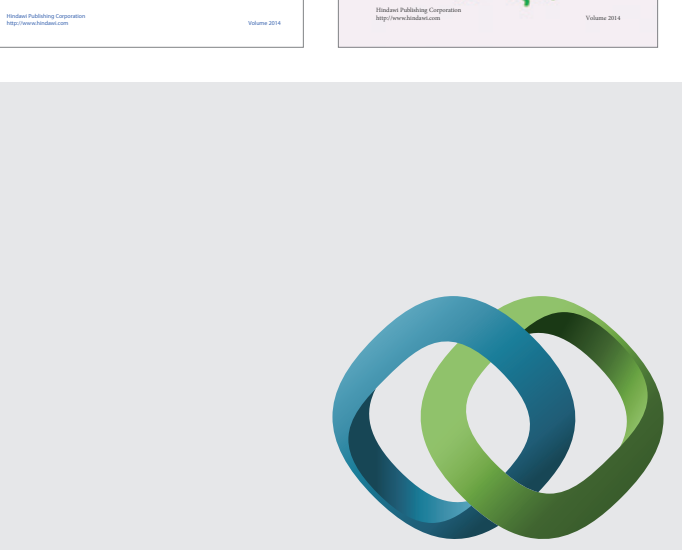

\section{Hindawi}

Submit your manuscripts at

http://www.hindawi.com
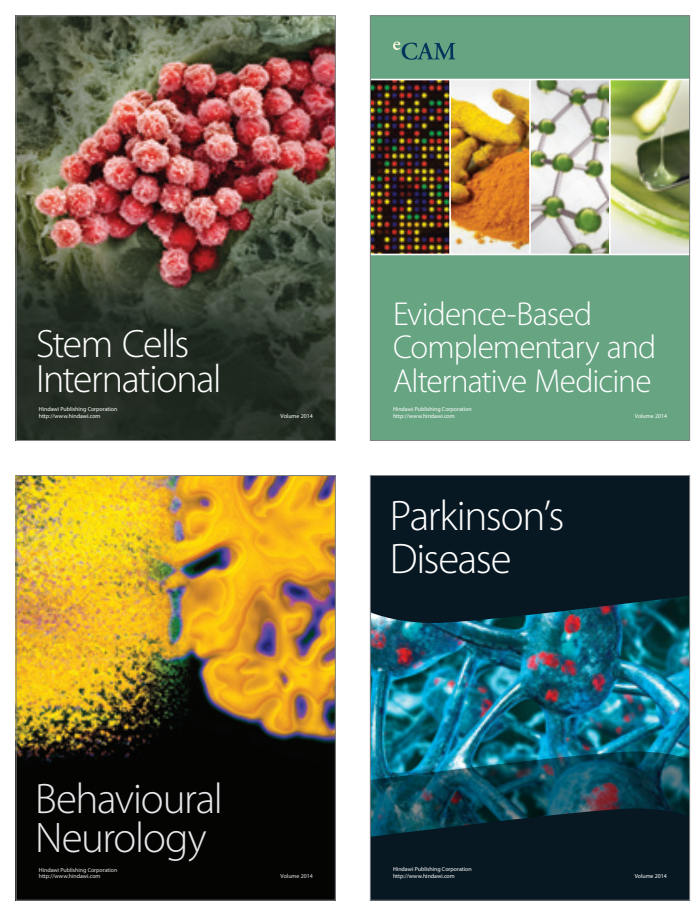

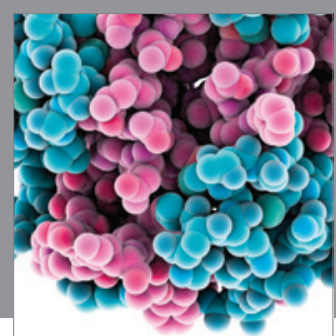

Journal of
Diabetes Research

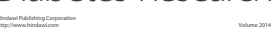

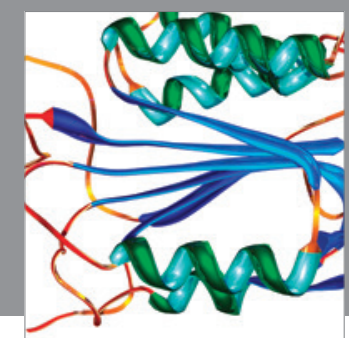

Disease Markers
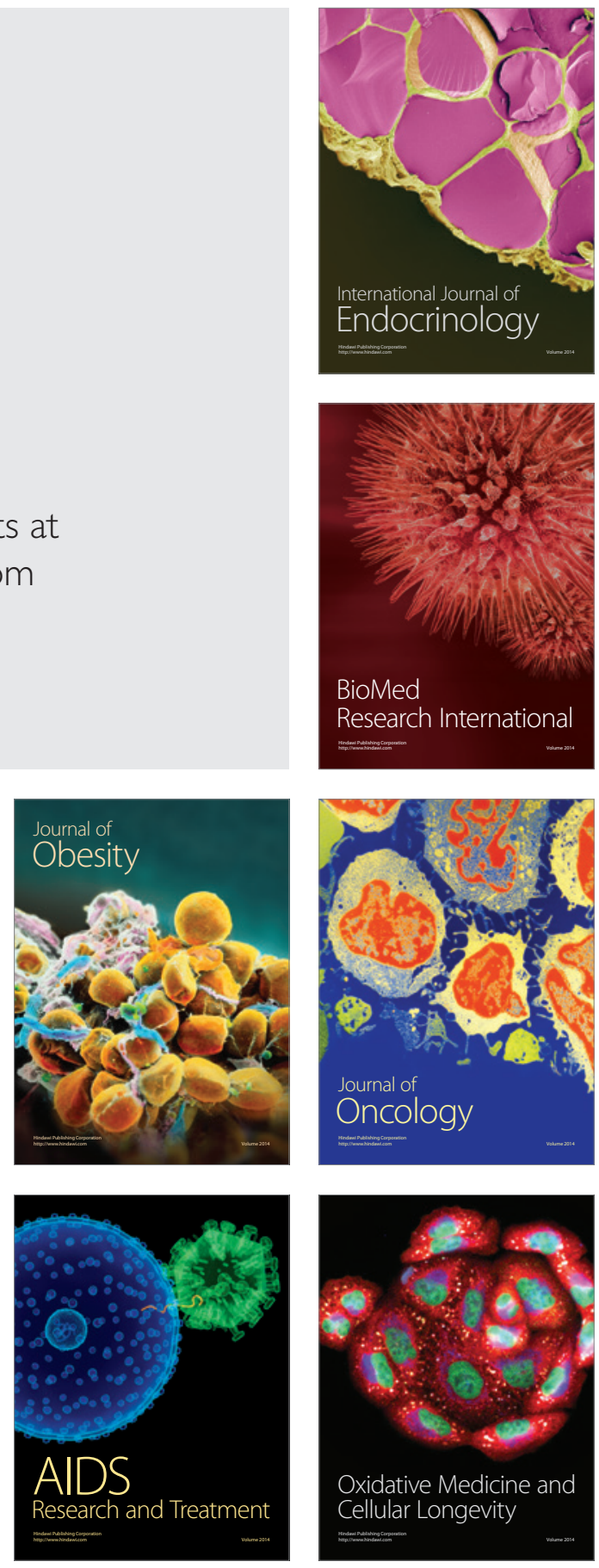